\title{
$1 \quad$ DCP2 plays multiple roles during Drosophila development - possible case of moonlighting?
}

2 Rohit Kunar and Jagat K Roy*

3 Cytogenetics Laboratory, Department of Zoology, Institute of Science, Banaras Hindu University,

4 Varanasi - 221005, Uttar Pradesh, India

5 *Address for Correspondence -

6 Jagat K Roy,

7 Cytogenetics Laboratory,

8 Department of Zoology,

9 Institute of Science,

10 Banaras Hindu University,

11 Varanasi - 221005,

12 Uttar Pradesh, India.

13 Fax: +91-542-236-8457

14 E-mail: jkroy@bhu.ac.in

15

16 Running title - DCP2 expression in Drosophila development 


\section{DCP2 plays multiple roles during Drosophila development - possible case of moonlighting?}

Rohit Kunar and Jagat K Roy*

Cytogenetics Laboratory, Department of Zoology, Institute of Science, Banaras Hindu University, Varanasi - 221005, Uttar Pradesh, India

\section{Abstract}

mRNA decapping proteins (DCPs) are components of the P-bodies in the cell which are hubs of mRNAs targeted for decay and they provide the cell with a reversible pool of mRNAs in response to cellular demands. The Drosophila genome codes for two decapping proteins, DCP1 and DCP2 out of which DCP2 is the cognate decapping enzyme. The present endeavour explores the endogenous promoter firing, transcript and protein expression of DCP2 in Drosophila wherein, besides a ubiquitous expression across development, we identify active expression paradigm during dorsal closure and a plausible moonlighting expression in the Corazonin neurons of the larval brain. We also demonstrate that the ablation of $D C P 2$ leads to embryonic lethality and defects in vital morphogenetic processes whereas a knockdown of $D C P 2$ in the Corazonin neurons reduces the sensitivity to ethanol in adults, thereby ascribing novel regulatory roles to DCP2. Our findings unravel novel putative roles for DCP2 and identify it as a candidate for studies on the regulated interplay of essential molecules during early development in Drosophila, nay the living world.

Keywords - Corazonin/ DCP2/ development/ epithelial morphogenesis/ ethanol sedation

\section{Introduction}

Organismal development mimics an orchestra with precisely timed and fine-tuned role(s) for each of the players. Balanced expression of genes requires timed activity of gene promoters at the proper site along with orderly degradation of transcripts and/or proteins (Yao and Ndoja, 2012; Ding, 2015). Decay of transcripts is one of the strategies to regulate gene expression (Ghosh and Jacobson, 2010) and the mRNA decapping proteins (DCPs) assume prime importance therein. These proteins initiate degradation of the mRNAs in cytoplasmic foci known as P-bodies, by removal of the 7-methylguanosine cap at the 5' end of the mRNAs (Coller and Parker, 2004). The Drosophila genome codes for two mRNA decapping proteins, viz., DCP1 and DCP2, out of which DCP2 is the cognate decapping protein. While DCP1 functions to activate DCP2 (Ren et al, 2012) and P-bodies/DCP1 have been implicated in miRNA mediated gene silencing (Rehwinkel et al, 2005), localization of the oskar mRNA in the Drosophila oocyte (Lin et al, 2006) and in oogenesis (Lin et al, 2008), DCP2 has been implicated in chronic nicotine induced 
locomotor hyperactivity in Drosophila (Ren et al, 2012). However, characterization of the role of decapping proteins in development has been limited to Arabidopsis (Xu et al, 2006) and Caenorhabditis elegans (Lall et al, 2005) only. Despite being the cognate decapping protein in Drosophila, the spatiotemporal dynamics of $D C P 2$ activity remains unexplored. The gene in Drosophila is $\sim 8 \mathrm{~kb}$ in length, has two curated promoters, viz., a proximal promoter, DCP2_1 and a second, downstream promoter, DCP2_2 (Eukaryotic Promoter Database, EPD; Dreos et al, 2014) and codes for four transcripts (FlyBase; Drysdale, 2008). Herein, we have tried to explore the temporal activity of the $D C P 2$ promoter using the conventional UAS-GAL4 system (Brand and Perrimon, 1993) wherein, we used a GAL4 driven by the $D C P 2$ promoter (DCP2 ${ }^{\text {GALA }}$; Lukacsovich et al, 2001; Ren et al, 2012) and combined it with a modified UAS line (G-TRACE; Evans et al, 2009) to delineate the real-time promoter activity of DCP2 during embryonic dorsal closure and in the larval tissues. In parallel, we endeavored to delineate the expression of the transcript isoforms or splice variants generated, and the expression paradigm of the translated protein. Although, $D C P 2$ is highly active and ubiquitous, high expression of the DCP2 protein was observed in the Corazonin $(\mathrm{Crz})$ neurons of the larval brain and renders the individuals less sensitive to ethanol when knocked down in the corazonin neurons and it is expressed along the A-P and D-V axes in the larval wing pouch. Loss-of-function mutants of $D C P 2$ are embryonic lethal and showed defects in epithelial morphogenesis and organization of the embryonic nervous systems along with elevation and spatial disruption of the JNK cascade. Collectively, our observations present us with a stage for further exploration of hitherto undescribed facets of $D C P 2$ activity and identify DCP2 as a potential candidate for explication of molecular interplay during Drosophila development.

\section{Materials and Methods}

\section{Fly strains, genetics and lethality assay}

All flies were raised on standard agar-cornmeal medium at $24 \pm 1^{\circ} \mathrm{C}$. Oregon $R^{+}$was used as the wild type control. For targeted gene expression (Brand and Perrimon, 1993), DCP2 $2^{B G 01766} / T M 3$, Ser $^{l}\left(D C P 2^{G A L A}\right.$; Ren et al, 2012), CCAP-GAL4, TH-GAL4, Ap-GAL4, Ddc-GAL4, UAS-GFP, UAS-mCD8::GFP and UAS$D C P 2^{R N A i}$ were obtained from the Bloomington Drosophila Stock Centre, while G-TRACE (Evans et al, 2009) was a kind gift from Prof. Utpal Banerjee, MBI, UCLA. DCP2 $2^{e 00034} / T M 3$, $\mathrm{Ser}^{l}$ was obtained from 
$D C P 2^{B G 01766} / T M 3, \operatorname{Ser}^{I}$ and $D C P 2^{e 00034} / T M 3, \operatorname{Ser}^{l}$ were further introgressed with TM3, ActGFP, $\mathrm{Ser}^{1} / T M 6 B$ stock in order to generate $D C P 2^{B G 01766} / T M 3, A c t G F P, \operatorname{Ser}^{1}$ or DCP2 $2^{e 00034} / T M 3$, ActGFP, Ser ${ }^{1}$ stocks. TRE-JNK (Chatterjee and Bohmann, 2012) was introgressed with $S p / C y O ; D C P 2^{B G 01766} / T M 3$, ActGFP, $\mathrm{Ser}^{l}$ or and Sp/CyO; DCP2 $2^{e 00034} / \mathrm{TM} 3, \mathrm{ActGFP}, \mathrm{Ser}^{l}$ to obtain TRE-JNK; DCP2 $2^{B G 01766} / T M 3$, ActGFP, Ser ${ }^{l}$ and TRE-JNK; DCP2 $2^{e 00034} / T M 3, A c t G F P, \operatorname{Ser}^{l}$ stocks, respectively.

For behaviour assays, $C r z-G a l 4 / C y O$ flies were crossed to $w^{1118}$ or $U A S-D C P 2^{R N A i}$ flies to generate $C r z-$ Gal4/+ (Control) or Crz-Gal4/+; UAS-DCP2 $2^{R N A i} /+$ (Experimental) genotypes.

Embryonic lethality was calculated as described in Bhuin and Roy, 2009. 100 embryos were transferred to agar plates and incubated for 24 to $48 \mathrm{~h}$ at $23^{\circ} \mathrm{C}$ and the total number of dead embryos was counted against total number of fertilized eggs. These fertilized eggs include the dead as well as the hatched embryos. The percentage of lethality was calculated as -

(No. of dead embryos/No. of fertilized embryos) $\times 100 \%$

The percentage (\%) lethality for each cross was calculated in triplicates and the mean lethality so obtained was tabulated and graphically represented using MS-Excel-2010. The final percentages have been calculated by multiplying the lethality obtained in every cross scheme with the inverse of the fraction of the progeny determined by standard Mendelian ratios.

\section{Detection of DCP2 transcript expression and analysis of splice variants}

Detection of transcript expression from $D C P 2$ was performed by reverse-transcriptase polymerase chain reaction (RT-PCR) using a combination of primers designed such that the amplicon size would discriminate between the individual isoforms which included a single reverse primer, DCP2_DBAE_R, which could bind to all of the transcripts, and two forward primers, viz., DCP2_BAE_F which would bind to isoforms DCP2-RA, RB and RE, and DCP2_D_F, which would bind to DCP2-RD. Being similar in architecture, DCP2-RA and RE would yield similar sized amplicons with the above primer pair, but DCP2-RD would yield a smaller amplicon. However, the 3'UTR is longer and unique for DCP2-RE and we exploited the architectural bias to discriminate between the two isoforms by designing an additional primer pair which would amplify the 3'UTR sequence of DCP2-RE uniquely. The table below (Table 1) shows the primer sequences, the combinations and the calculated amplicon sizes for each of the isoform with each of the primer pairs. The unique amplicons are italicized. RT-PCR was performed according to Lakhotia et al, 2012 in the samples discussed in the results section. 
Table 1: List of primer sequences, combinations generated and calculated amplicon sizes for detection of expression of $D C P 2$ transcript isoforms

\begin{tabular}{|c|c|c|c|c|c|}
\hline \multirow{2}{*}{ Primer Pair } & \multirow{2}{*}{ Sequence $\left(5^{\prime}-3^{\prime}\right)$} & \multicolumn{4}{|c|}{ Amplicon size(in base pair) } \\
\hline & & $\mathrm{RD}$ & $\mathrm{RB}$ & RA & $\mathrm{RE}$ \\
\hline \multirow{2}{*}{$\begin{array}{l}\mathrm{DCP} 2{ }^{\mathrm{D}} \mathrm{F} \\
\mathrm{DCP} 2{ }_{\mathrm{DBAE}} \mathrm{R}\end{array}$} & ACAACGATTCAATACATATACAGCT & \multirow{2}{*}{165} & \multirow{2}{*}{-} & \multirow{2}{*}{-} & \multirow{2}{*}{-} \\
\hline & CTGTTTTTGTTGCTCGTGTTGT & & & & \\
\hline \multirow{2}{*}{$\begin{array}{l}\mathrm{DCP} 2 \mathrm{BAE}^{\mathrm{F}} \\
\mathrm{DCP} 2 \mathrm{DBAE}_{\mathrm{R}}\end{array}$} & GCAATTTAGATCGCGAAAAAGTTC & \multirow{2}{*}{-} & \multirow{2}{*}{159} & \multirow{2}{*}{974} & \multirow{2}{*}{974} \\
\hline & CTGTTTTTGTTGCTCGTGTTGT & & & & \\
\hline \multirow{2}{*}{$\begin{array}{l}\text { DCP2 } 2 E U-F \\
\text { DCP2 } E U R\end{array}$} & TCATTTGTCTGGGCCAAGTGAC & \multirow{2}{*}{-} & \multirow{2}{*}{-} & \multirow{2}{*}{-} & \multirow{2}{*}{233} \\
\hline & TGGGATTGCAGTTCATCAAATG & & & & \\
\hline
\end{tabular}

\section{Embryo collection and fixation}

112 All flies were made to lay eggs on standard agar plates supplemented with sugar and propanoic acid and 113 eggs were collected according to Narasimha and Brown, 2006, with slight modifications. For whole 114 mount preparations and immunostaining of embryos, different alleles and transgenes were balanced with 115 GFP tagged balancers and only non-GFP or driven embryos were selected for experimental purpose. 116 Embryo staging was done according to Hartenstein's Atlas of Drosophila Development, 1993.

\section{Immunocytochemistry}

Drosophila embryos were fixed and imaged as described by Narasimha and Brown, 2006. The dechorionated and devitellized embryos were fixed in $4 \%$ para-formaldehyde solution and stored in absolute methanol. Immunostaining of the embryos was done as described in Nandy and Roy, 2019. Late third instar larval tissues were dissected out in 1X PBS, fixed in 4\% paraformaldehyde for 20 min at RT and immunostained as described previously in Banerjee and Roy, 2017. The primary antibodies used were - mouse anti-DCP2 (1:50; PCRP-DCP2-1D6, DSHB), mouse anti-Fasciclin II (1:100; 1D4, DSHB), mouse anti-Fasciclin III (1:100; 7G10, DSHB) and rabbit anti-phospho-JNK/SAPK (1:100; 81E11 Cell

125 Signaling Technology). Appropriate secondary antibodies conjugated either with Cy3 (1:200, Sigma126 Aldrich, India) or Alexa Fluor 488 (1:200; Molecular Probes, USA) or Alexa Fluor 546 (1:200; Molecular 127 Probes, USA) were used to detect the given primary antibody, while chromatin was visualized with DAPI 128 (4', 6-diamidino-2-phenylindole dihydrochloride, $1 \mu \mathrm{g} / \mathrm{ml}$ Sigma-Aldrich). For imaging of live embryos 129 for real-time promoter analysis using G-TRACE or JNK signaling, embryos of the desired genotype were 130 rinsed in 1 X PBS, dechorionated in bleach and mounted in halocarbon oil and observed directly. 
Cuticle preparations were made from embryos as described by Wieschaus and Nusslein-Volhard, 1986 along with some modifications as described in Sasikumar and Roy, 2008. Briefly, the eggs were dechorionated in bleach and washed in an aqueous solution containing $0.7 \% \mathrm{NaCl}$ and $0.02 \%$ Triton-X 100. The eggs were washed thrice in $0.1 \%$ Triton-X, devitellinised in a mixture of methanol and $n-$ heptane $(1: 1 \mathrm{v} / \mathrm{v})$ mixture. They were fixed in 1 part glycerol - 4 parts acetic acid for $1 \mathrm{~h}$, mounted in Hoyer's mountant and cleared at $60^{\circ} \mathrm{C}$ overnight.

\section{Microscopy and Documentation}

The immunostained slides were observed under Zeiss LSM 510 Meta Laser Scanning Confocal microscope, analysed with ZEN12 and LSM softwares and assembled using Adobe Photoshop 7.0. The cuticles were observed in dark field or phase-contrast optics, namely 10X Plan Fluor Ph1 DLL (0.3 NA), 20X Plan Fluor Ph1 DLL (0.5 NA) and 40X Plan Fluor Ph2 DLL (0.75 NA) objectives (Nikon, Japan) and the images were captured with a Nikon Digital camera DXM1200. Fluorescence imaging of embryos for analysis of $D C P 2$ promoter using G-TRACE or JNK signaling was done in Nikon 90i Fluorescence microscope under 10X Plan Fluor Ph1 DLL (0.3 NA), 20X Plan Fluor Ph1 DLL (0.5 NA) objectives.

\section{Behaviour Assays}

Groups of 20 males and females (1-3 days old) of the desired genotypes, viz., Crz-Gal4/+ (Control) and $\mathrm{Crz}-\mathrm{Gal} / \mathrm{A}+\mathrm{UAS}-\mathrm{DCP} 2^{R N A i} /+$, maintained on food vials in a $12 \mathrm{~L}: 12 \mathrm{D}$ conditions at $23^{\circ} \mathrm{C}$ for 1 day were used for the following behaviour assays.

\section{Ethanol Sedation Assay}

Ethanol sedation assays were performed as described previously (McClure et al, 2013) with minor alterations. Briefly, flies were transferred to empty vials, sealed with cotton plugs and allowed to acclimatize for 10-20 min. The cotton plugs were replaced with fresh plugs containing $1 \mathrm{ml}$ of $100 \%$ ethanol. They were maintained in such "booze chambers" for 15-20 mins. During the treatment, files were mechanically stimulated by tapping and/or mechanically swirling the vials at intervals of 5 mins. Flies able to climb the walls and/or move their appendages on the floor of the vial were considered "nonsedated" while those unable to execute such activity were considered "sedated". The number of sedated flies was counted at 5 min intervals. The time to 50\% sedation (ST50) was determined by manual extrapolation.

\section{Recovery from Ethanol Sedation}


Recovery from ethanol induced sedation was assayed as described by Sha et al, 2014. Following exposure to ethanol (described above), the cotton plugs were replaced with fresh plugs and the vials were inverted to place them upside down. The number of "non-sedated" flies (considered as "recovered") was counted every $10 \mathrm{~min}$.

\section{Results and Discussion}

\section{DCP2 mRNAs are expressed ubiquitously throughout Drosophila development}

In order to determine the presence or absence of DCP2 at a particular stage alongwith identification of the exact isoform(s)/splice variant(s) expressed therein, RT-PCR was performed using primers designed for the same. DCP2 expression was detectable at all stages of development, viz. embryo (0-24h), larval stages $\left(1^{\text {st }}, 2^{\text {nd }}\right.$ and $3^{\text {rd }}$ instars $)$, pupal and adult stages. Among the four annotated variants, DCP2_RE (FBtr0304975) and DCP2_RA (FBtr0075538) was observed in all stages of fly development, whereas DCP2_RD (FBtr0100528) was observed only in the larval gonads, viz. testes and ovaries, and in the adult flies (Figure 1). The other variant, DCP2_RB (FBtr0075539) was detectable only in the pupae and adults but was absent in larval stages. Out of the four isoforms however, DCP2_RA and DCP2_RE is observed to be expressed throughout development but DCP2_RE appears to be the most abundant and ubiquitous isoform expressed. Although, DCP2-RB is driven by the same promoter which drives DCP2_RA and RE, its absence does not necessarily indicate dearth of expression. In-silico analyses and data mining from the Eukaryotic Promoter Database (Dreos et al, 2014, 2017) indicate that DCP2-RD may be driven by the second promoter of DCP2 (DCP2_2). The protein isoforms coded by DCP2_RB and DCP2_RD are identical in sequence and size, but the exclusive expression of DCP2_RD in the larval gonadal tissues (ovaries and testes) and at a very low titre in the adults may be owing to the promoter being responsive to transcription factors in the gonadal tissues only and/or a putative undiscovered function of the transcript therein, despite absence of visible quantities of DCP2_RB.

\section{$D C P 2$ expresses in cells of diverse developmental lineages in the Drosophila embryo and the $D C P 2$} promoter vis-à-vis $D C P 2$ is active since early development

Evolution has been parsimonious in designing genes and ascribing roles to them and hence, determination of gene functions becomes incomplete without identification of the expression dynamics of the gene. In order to determine the endogenous expression pattern of DCP2 in Drosophila melanogaster, we used the $D C P 2^{G A L 4}\left(D C P 2^{B G 01766}\right)$ which has a P $\{\mathrm{GT} 1\}$ construct (Lukacsovich et al, 2001) bearing a GAL4, immediately downstream to the $D C P 2$ promoter. Using GFP as a reporter, we detected extremely robust signal in the late embryonic stages, wherein it expresses strongly in the embryonic epithelia (ectoderm) (Fig. 2A), the central nervous system (neuro-ectoderm) (Fig. 2B) and the dorsal muscles (mesoderm) 
(Fig. 2C) and is uniformly ubiquitous in all the segments in the embryo. With such a robust expression (of GFP), which is actually driven by the $D C P 2$ promoter, it is evident that $D C P 2$ is expressed and is active in embryonic cells derived from differing developmental lineages.

Dorsal closure is a major morphgenetic event during embryonic developemnt in Drosophila (MartinezArias, 1993) and involves an orchestrated interplay of numerous molecules (Lada et al, 2012) to drive the concerted movement of lateral epithelial cell sheets. With $D C P 2$ being expressed strongly in the embryonic epithelium, we investigated the possibility and nature of activity of the $D C P 2$ promoter during dorsal closure. To determine the real-time activity of the $D C P 2$ promoter during dorsal closure, we used a GAL4-responsive tripartite construct, G-TRACE (Evans et al, 2009). Using this transgenic line, in the embryonic stages (Figure 3), we observed that DCP2 expresses in a more or less ubiquitous pattern very early in development, even prior to Stage 10 (Figure 3A). However, real-time expression was not detected in Stage 10, in which the germband is fully extended (Figure 3B and 3D), and the Stage 12, wherein the germband is fully retracted (Figure 3J and 3L). In the intervening stage, wherein the germband starts retracting, i.e., Stage 11, we detect strong expression of $D C P 2$ (Figure 3F and 3H). Again, when the epithelial sweeping initiates following germband retraction (Stage13), we see a surge in the RFP expression (Figure 3N and 3P) which intensifies further in Stage 14, in which the lateral epithelia on either side are still moving (Figure 3R and 3T). This intense RFP expression is visible in Stage 16 as well (Figure 3V and 3X). The Stages 10 and 12 are developmental periods of low cell migration as against Stages 11, 13 and 14 wherein the epithelium moves as an initiative of collective cell migration and coordinated cell-shape changes. The expression potential of the $D C P 2$ promoter across DC revealed expression "crests and troughs", such that the "crests" paralleled the periods in which cellular mobility or migration was maximal and vice-versa (Figure 3Y). The RFP activity is detectable only in stages which involve collective cell movement. The eGFP expression however depicts an early initial pulse of the gene expression which plausibly maintains a basal level of gene product. Hence, the dynamics of the promoter reflects a tightly regulated expression of $D C P 2$ and brings to light that $D C P 2$ may be an essential player during collective cell migration vis-à-vis epithelial morphogenesis.

\section{DCP2 expresses in the amnioserosa and lateral epithelium during dorsal closure and its loss affects survival, epithelial morphogenesis and development of nervous system in the Drosophila embryo}

Since $D C P 2$ shows active expression paradigms during embryonic dorsal closure (Figure 9), we examined the expression of DCP2 in the lateral epithelia in Stage 13 embryos along with the expression of activated JNK, a key mediator of dorsal closure (Jacinto et al, 2002), and Fasciclin III, a cell adhesion molecule (Bahri et al, 2010), both of which are expressed at the lateral epithelia and the leading edge (LE) 
cells. DCP2 was found to be expressed in the amnioserosa and throughout the lateral epithelium as well as in the cells at the LE (Figure 4). During the later stages of dorsal closure, parallel to the contra-lateral movement of the epithelia towards the dorsal side, the axon pathways are pioneered in the CNS across the ventral nerve cord, which form the complete nervous system by the end of Stage 16 (Bhuin and Roy, 2009). Examination of the ventral nerve cord also showed strong cytoplasmic expression of DCP2 (Figure 5).

We found that embryos homozygous for loss-of-function alleles of $D C P 2$ (viz., $D C P 2^{B G 01766}$ and $\left.D C P 2^{e 00034}\right)$ show embryonic lethality. $D C P 2^{B G 01766}$ homozygotes are $100 \%$ embryonic lethal $(\mathrm{N}=500)$ whereas $D C P 2^{e 00034}$ homozygotes show $12 \%$ lethality $(\mathrm{N}=500)$ at the embryonic stage and the remaining die before reaching the second instar larval stage.

\section{Defects in Epithelial Morphogenesis}

Since we found strong expression of $D C P 2$ in the embryonic epithelium, we endeavored to explore whether a loss of $D C P 2$ function affects epithelial morphogenesis. Analysis of embryonic cuticles showed that all mutants displayed pronounced defects in epithelial morphogenesis patterns, ranging from defects in size, viz., anterio-posterior or dorso-ventral dimensions, head involution defects and morphological defects, viz., u-shaped or puckering (Figures 6). Since these defects are not mutually exclusive in that, a single mutant embryo could be displaying multiple defects at the same time, the morphological aberrations were scored individually first and then analysed for the presence of other concomitant defects. While $82.6 \%$ of the $D C P 2^{B G 01766}$ homozygotes show altered anterio-posterior or dorso-ventral dimensions (viz., elongated or compressed) of which $68.4 \%$ embryos are defective in head involution and $36.8 \%$ embryos are puckered, $21.7 \%$ embryos have gross defects in all the morphological parameters analysed. $4.3 \%$ of the embryos are exclusively puckered and $6.5 \%$ embryos show defects in head involution only. None of the $D C P 2^{e 00034}$ homozygotes analysed was exclusively puckered. $80 \%$ of the embryos observed show altered dimensions out of which $12.5 \%$ are puckered and are defective in head involution and 62.5\% embryos are not puckered but show head involution defects. $20 \%$ of the embryos observed show defects exclusively in dimensions or head involution. Figure 7 shows the above data represented with the help of a Venn diagram.

\section{Defects in Nervous System development}

We used mAbBP102, an antibody to mark all CNS axons (Seeger et al. 1993) such that the gross morphology of CNS in an embryo is revealed. In wild-type embryos, axons form an orthogonal structure having longitudinal axon tracts. These axon tracts run anterio-posteriorly, being positioned at either side 
of the midline, and a pair of commissural tracts joins the longitudinal pathways in each segment of the embryo (Bhuin and Roy, 2009). DCP2 $2^{B G 01766}$ homozygotes showed thinning of longitudinal connectives and compressed segmental commissures (Figure 8 B and B') similar to the karussell phenotype (Hummel et al, 1999), whereas, DCP2 $2^{e 00034}$ homozygotes showed thinning of longitudinal connectives and lateral commissures (Figure $8 \mathbf{C}$ and $\mathbf{C}^{\prime}$ ).

In order to study the embryonic PNS axons further, mutant embryos were stained with mAb22C10, which recognizes the microtubule-associated protein, futsch (Hummel et al. 2000). It labels all the cell bodies, dendrites, and axons of all PNS neurons, and a subset of neurons in the VNC of the CNS (Fujita et al. 1982). Therefore, defects, such as the disruption of the nervous system, the collapse of the axon tracts, fasciculation defects/thinning of axons, and the loss or gain of neurons can often be distinguished. In the wild type embryos, each segment contains three highly stereotyped clusters of PNS neurons connected by axon bundles. In the mutants, misrouting of axons and collapsed axons could be detected (Fig. 8 E, E' and $\left.\mathbf{F}, \mathbf{F}^{\prime}\right)$, which were absent in the wild type, implying a role for DCP2 in the fasciculating axons.

\section{DCP2 loss-of-function mutants show elevation and spatial perturbation of JNK signaling}

The JNK signaling cascade is an essential player of Drosophila gastrulation vis-à-vis embryonic development wherein it modulates important events such as dorsal closure (Jacinto et al, 2002; Kushnir et al., 2017) and architecture of the nervous systems (Shklover et al, 2015; Karkali et al, 2016). Since DCP2 loss-of-function mutants show defects associated with either process, we wanted to investigate the spatial expression of the JNK cascade. We harnessed the bio-sensor, TRE-RFP to identify the spatial pattern of JNK signaling (Chatterjee and Bohmann, 2012) in the wild type embryos and in embryos homozygous for loss-of-function alleles of $D C P 2$. Both $D C P 2^{B G 01766}$ and $D C P 2^{e 00034}$ homozygotes showed enhanced RFP

277 expression, implying an elevation in the JNK signaling cascade. Further, the pattern of RFP vis-à-vis JNK 278 signaling is spatially disrupted in the DCP2 mutants, implying a perturbation and/or misregulated JNK 279 signaling (Figure 9). In the wild type embryos it is expressed at the juncture of the two epithelial sheets following completion of dorsal closure, mimicking the stitch at a suture, whereas the mutant homozygotes show a spatial distortion of JNK signaling.

282 In the developing Drosophila embryo undergoing gastrulation, epithelial morphogenesis and axonogenesis are morphogenetic events of utmost importance that require a well orchestrated spatiotemporal regulation of gene expression. During initiation of dorsal closure wherein the two lateral epithelia initiate contra-lateral movement to eventually seal the dorsal opening, the dorsal-most lateral epithelial cells express high levels of JNK (Noselli and Agnes, 1999). The JNK signaling pathway is a 
(Noselli, 1998; Noselli and Agnes, 1999; Ramet et al 2002; Stronach and Perimon, 2002).While DCP2 co-expresses with JNK ubiquitously on the dorso-lateral epithelia, the leading edge and the amnioserosa, monitoring the activity of the $D C P 2$ promoter in real time across the stages of dorsal closure shows spurts of promoter firing in the stages which involve large scale cell migration and movement. Such subtle and precisely timed gene activity is indicative of thorough fine-tuning of the expression of $D C P 2$. The ablation of $D C P 2$ does not lead to "dorsal open" embryos, but generates a spectrum of defects including altered embryonic dimensions and defects in head involution, improper fasciculation of axons and defects in segmental commissures and longitudinal connectives, causes embryonic and larval lethality implies significant perturbation in these developmental gene expression programs and indicate a more concerted and fundamental role of $D C P 2$ in regulating these phenomena. It is worth mentioning that despite ubiquitous expression of $D C P 2$, the ectodermal (epithelium) and neuro-ectodermal (CNS and PNS) tissues are most affected following ablation. It is interesting to note that while the nematode worm is a closer relative of the fly in the evolutionary tree, the embryonic lethality and the developmental defects are similar to those observed in a distant relative, Arabidopsis (Xu et al, 2006; Goeres et al, 2007). Since the JNK signaling pathway is fundamental to the process of dorsal closure during gastrulation in Drosophila embryos (Noselli, 1998; Noselli and Agnes, 1999; Ramet et al, 2002; Stronach and Perrimon, 2002) and both epithelial morphogenesis and CNS development are dependent on JNK activity (Jacinto et al, 2002; Kushnir et al., 2017; Shklover et al, 2015; Karkali et al, 2016), the altered expression patterns of JNK or misdirected JNK signaling under the influence of loss of DCP2 in the different alleles could be a probable cause of the defects observed in each case.

The $D C P 2$ promoter shows consistent developmental activity in the larval tissues along with tissuespecific expression paradigms of the translated protein

Real-time activity of the promoter in the various larval tissues, with a better insight, demonstrates that despite expression since early development, the $D C P 2$ promoter is active during late stages of larval development as well. In the larval tissues (118 $\pm 1 \mathrm{~h} \mathrm{ALH})$, we could identify a consistent GFP expression in the larval brain, eye-antennal disc, salivary gland and wing discs. Besides prior developmental activation and ubiquitous expression, the $D C P 2$ promoter shows enhanced activity/expression in specific cells in the brain (Figure $10 \mathbf{B}$ and $\mathbf{C}$ ) and eye disc (Figure 10 G and $\mathbf{H}$ ). Although, the ventral ganglion depicts an overlap of prior and real-time activity (Figure 10 B-D), the cerebral hemispheres show selective expression in real-time, which is limited to cells of the antennal lobe and the Kenyon cells

318 (Figure 10 B'-D'). Similarly, the cells in constituting the antennal disc show a more consistent DCP2 activity, exemplified the greater degree of overlap of the reporters (Figure 10 G-I). However, the cells of the eye disc show heterogeneity of reporter expression, viz., DCP2 is active in all the ommatidia but 
321

322

323

324

325

326

327

328

329

330

331

332

333

334

335

336

337

certain cells show a transient activity at the stage observed (Figure 10 G'-I'). While the salivary gland nuclei show a complete colocalization of reporters with similar intensity (Figure 10 L-N), wing discs show a lower expression of eGFP as compared to RFP.

Since, the GAL4 is driven by the de novo promoter of $D C P 2$, which in absence of the GAL4 coding region, would have transcribed the gene per se, this transgenic construct, viz., G-TRACE allows the spatio-temporal expression potential of the promoter to be determined. Thus, the expression of the reporters (GFP and RFP) may be directly correlated with the gene expression pattern or potential in the wild type individual and hence, via the GAL4, directly demonstrates the spatio-temporal gene expression dynamics.

\section{Brain}

In the larval brain, immunolocalisation of DCP2 shows a uniform cytoplasmic expression throughout the dorso-ventral and anterio-posterior axes of the tissue (Figure 11A', B' and D'). However, significantly high levels were detected in a subset of neurons in the ventral ganglion (Figure 11A' and D') and in a cluster of neurons in the dorso-lateral and dorso-medial region of the central brain (Figure 11A' and C'), which are proximal to the Mushroom Body as well as in the Kenyon cells (Figure 11K and O). However, it is completely absent from the most prominent neuronal structures viz., the Mushroom Body in the central brain and in the neurons of the optic lobe (Figure 11M-O).

\section{Salivary Glands}

In the salivary glands, DCP2 shows a punctuate distribution in the cytoplasm and decorates the nuclear and cellular membranes arduously (Figure 11E' and E"). The cytoplasmic vesicles appear bounded by bodies rich in DCP2 (Figure 11E"). Since DCP2 is a cognate resident of the P-bodies, it may be fair enough to interpret the cytoplasmic network of DCP2 punctae in the glands as the pattern of P-bodies which are essential for maintaining transcript homoeostasis.

\section{Wing discs}

The wing discs show very strong expression of DCP2 in the pouch region as compared with the notum (Figure 11F'), besides the uniform ubiquitous cytoplasmic distribution similar to that observed in other tissues. Most notably, the expression of DCP2 in the central sections of the pouch overlaps with the expression of the Anterio-Posterior determinant Decapentaplegic (Dpp) (Zecca et al, 1995) and the Dorso-Ventral determinant Wingless (Wg) (Neumann and Cohen, 1997), thereby presenting a 
“cruciform" pattern in the pouch (Figure 11G'), which may be essential during the morphogenesis of the wing blade.

Immunolocalisation of DCP2 to the cytoplasm in all the tissues examined across development recapitulates the results observed in similar studies in the nematode worm, C. elegans (Lall et al, 2005) and in the thale cress, Arabidopsis (Xu et al, 2006). In spite of uniform ubiquitous cytoplasmic expression in the larval tissues, certain paradigms of expression have been noticed. The protein shows distinct punctate expression of the protein in the wing imaginal disc along the anterio-posterior and dorso-ventral axes in the wing pouch, mimics the expression patterns of the TGF-beta homologue Decapentaplegic and Wingless, respectively. In the salivary glands as well, the protein is cytoplasmic but shows high titres at the membranes. Being the sole decapping agent in Drosophila, DCP2 is expressed ubiquitously throughout development but, the selectively high expression in certain cell types in the brain or the wing pouch point towards some yet unknown "moonlighting" functions of $D C P 2$ in the development and maintenance of cellular homoeostasis in these tissues.

\section{DCP2 shows high expression in the Corazonin neurons in the larval CNS}

364 Besides ubiquitous expression, DCP2 has a typical expression paradigm in a subset of neurons in the

365 larval CNS. In order to identify/type the DCP2 immunopositive neuron(s) in the larval ventral nerve cord (VNC), we tried mapping them against the Fasciclin II (FasII) landmark system (Santos et al, 2007) (Figure 12). Comparing the FasII "coordinates" with the DCP2 expression paradigm, we observed that DCP2 expresses in a cluster of three neurons in the Dorso-lateral (DL) region and in a neuron located medial to the DL neurons (Dorso-medial; DM) in the central brain, and in eight pairs of bilateral neurons in the ventral nerve cord. The neurons in the ventral ganglion correspond to a subset of the thoracic (T2 and T3) and abdominal (A1 - A6) neuromeres. Although DCP2 is absent from the most prominent neuronal structures expressing FasII, viz., the Mushroom Body and the neurons innervating the eye, in the central brain (Figure 12 D-F) and in the Dorso-lateral and Dorso-medial longitudinal tracts in the VNC

374 (Figure 12 G-I), the DL neurons appear to innervate the Ring gland and the aorta. Lateral views of the 375 central brain (Figure 12 D'-F') and the VNC (Figure 12 G'-I') show that the DCP2-positive neurons lie 376 below the Fas II immunopositive Dorso-lateral and Dorso-medial longitudinal tracts but ascend above the 377 Mushroom Body (MB) in the central brain.

378 While DCP2 did not show co-expression with the different neuropeptides viz. Crustacean Cardioactive 379 Peptide (CCAP; Veverytsa and Allan, 2012), Drosophila Insulin like Peptide-2 (Dilp2; Liu et al, 2016), 380 Corazonin (Crz; Lee et al, 2008) and short Neuropeptide F (sNPF; Nassel et al, 2008), biogenic amines 
Wegener, 2008), or the transcription factor Apterous (Ap; Rincon-Limas et al, 1999) (Supplementary

Figure 2), complete colocalization or overlap of expression was observed with the neurons expressing Corazonin (Figure 13), while only the Dorso-lateral neurons showed co-expression with sNPF (Supplementary Figure 3). Corazonin neurons constitute three neuronal subsets, viz., the dorso-lateral (DL) and dorso-medial Crz neurons (DM), and the Crz neurons in the ventral nerve cord (vCrz) (Lee et al, 2008), are essential for combating stress (Zhao et al, 2010) and co-express the transcription factor Apontic, which is necessary and sufficient to mediate sensitivity to ethanol (McClure, 2013).

\section{Knockdown of $D C P 2$ in the Corazonin neurons reduces sensitivity to Ethanol}

We further asked whether $D C P 2$ function in the Corazonin neurons is required for their activity. When $D C P 2$ was knocked down in these neurons specifically, it did not affect the morphology, pathfinding or architecture of the Crz neurons in the larval brain (Supplementary Figure 4) but, delayed and/or reduced the sedation sensitivity to ethanol in the adult flies. The time to 50\% sedation (ST50) was calculated to be $\sim 8.5 \mathrm{~min}$ for the control flies ( $\mathrm{N}=200$ ), while the DCP2 knocked down flies showed an ST50 of $\sim 13.5$ $\min (\mathrm{N}=200)$. While the control flies are sedated completely in $\sim 12 \mathrm{~min}$, the DCP2 hypomorphs are active till $\sim 19$ min (Figure 14 A and B). This demonstrates the reduced sensitivity of the Crz GALA>UAS DCP2 RNAi knocked down flies to ethanol.

Following sedation, the DCP2 hypomorphs showed early onset of recovery ( 30 min) as against the control flies, which started showing activity/onset of recovery after $\sim 110 \mathrm{~min}$. While the control flies started assuming normal standing posture in $\sim 2 \mathrm{~h}$, the $D C P 2$ knocked down flies showed significant early recovery, with $\sim 80 \%$ of the flies recovering by $3 \mathrm{~h}$ as against $\sim 40 \%$ recovery exhibited by the control flies in the same time (Figure $14 \mathbf{C}$ and D). Also, sedation induced death was higher in the control group as compared to the $D C P 2$ hypomorphs. During sedation and recovery phases, no sex specific differences were observed in flies of either genotype. These results suggest that $D C P 2$ function is required in the $\mathrm{Crz}$ neurons for regulation of ethanol related behaviour and ethanol metabolism.

406 The Crz neurons require the transcription factor Dimmed and its target enzyme, Peptidylglycine-alpha407 hydroxylating monooxygenase (PHM) (Park et al, 2008) for synthesis of Corazonin while the 408 transcription factor Apontic (Apt) is necessary and sufficient for regulating the activity of the Crz neurons and/or release of Corazonin during ethanol exposure (McClure, 2013). The delay in sedatory behaviour during ethanol exposure and the quick recovery from sedation demonstrate impaired function of the $\mathrm{Crz}$ although the mechanism behind such altered physiology remains unknown. 


\section{Summary and Conclusion}

414 Analysis and identification of the expression patterns of genes and/or proteins in model organisms across

415 the evolutionary tree are important for understanding the spectral paradigm of gene function. The extent

416 to which a gene and its expressome are conserved across diverse organisms indicates the precision of its

417 function across phyla. mRNA decapping proteins are present in all metazoans and serve to initiate the

418 decay of mRNA and are therefore important for regulation of gene expression vis-à-vis cellular

419 physiology. The patterns of expression and paradigm range of physiological aberrations following the

420 ablation or knockdown of $D C P 2$ is indicative of the fundamental regulatory role played by it during

421 development and bring to light the hitherto undiscovered plausible novel functions of DCP2. It is yet

422 unknown as to whether it's function in the modulation of developmental events is via the de novo

423 function of mRNA decapping or is a manifestation of moonlighting behaviour (Mani et al, 2014) .

424 Summarizing the present observations, our findings demonstrate that $D C P 2$ plays a major modulatory

425 function in developmental gene expression and is essential for maintenance of organismal physiology at all stages of development.

\section{Acknowledgements}

429 The authors acknowledge the fly community for generously providing fly stocks. We thank Prof. B. J. 430 Rao, TIFR, Mumbai for providing the TRE-JNK/CyO stock, Prof. Gaiti Hasan, NCBS, Bangalore for providing the $s N P F-G A L 4$, Dilp2-GALA and $C r z-G A L 4 / C y O$ stocks and Prof. Utpal Banerjee, UCLA for providing the G-TRACE/CyO flies. We duly acknowledge the National Facility for Laser Scanning Confocal Microscopy, Department of Zoology, Banaras Hindu University. Financial support from DSTScience and Technology (DST) for providing INSPIRE Fellowship to RK.

\section{Author Contributions}

439 RK, conceptualization, resources, methodology, investigation, data curation, formal analysis and 440 interpretation, writing the manuscript. JKR, supervision, resources, analysis and interpretation, writing the 441 manuscript.

\section{Conflict of Interest}

443 The authors declare no conflict of interest. 


\section{References}

\section{6}

471 Friggi-Grelin, F., Coulom, H., Meller, M., Gomez, D., Hirsh, J. and Birman, S., 2003. Targeted gene 472 expression in Drosophila dopaminergic cells using regulatory sequences from tyrosine hydroxylase.

473 Journal of neurobiology, 54(4), pp.618-627.

474 Fujita, S.C., Zipursky, S.L., Benzer, S., Ferrus, A. and Shotwell, S.L., 1982. Monoclonal antibodies against 475 the Drosophila nervous system. Proceedings of the National Academy of Sciences, 79(24), pp.7929-7933.

476 Ghosh, S. and Jacobson, A., 2010. RNA decay modulates gene expression and controls its fidelity. Wiley 477 Interdisciplinary Reviews: RNA, 1(3), pp.351-361. 
478 Goeres, D.C., Van Norman, J.M., Zhang, W., Fauver, N.A., Spencer, M.L. and Sieburth, L.E., 2007. 479 Components of the Arabidopsis mRNA decapping complex are required for early seedling 480 development. The Plant Cell, 19(5), pp.1549-1564.

481

Hartenstein, V., 1993. Atlas of Drosophila development (Vol. 328). Cold Spring Harbor Laboratory Press.

Hummel, T., Krukkert, K., Roos, J., Davis, G. and Klämbt, C., 2000. Drosophila Futsch/22C10 is a MAP1B-like protein required for dendritic and axonal development. Neuron, 26(2), pp.357-370.

Jacinto, A., Woolner, S. and Martin, P., 2002. Dynamic analysis of dorsal closure in Drosophila: from genetics to cell biology. Developmental cell, 3(1), pp.9-19.

Karkali, K., Panayotou, G., Saunders, T.E. and Martin-Blanco, E., 2016. The JNK signaling links the CNS architectural organization to motor coordination in the Drosophila embryo.

Kushnir, T., Mezuman, S., Bar-Cohen, S., Lange, R., Paroush, Z.E. and Helman, A., 2017. Novel interplay between JNK and Egfr signaling in Drosophila dorsal closure. PLoS genetics, 13(6), p.e1006860.

Lada, K., Gorfinkiel, N. and Arias, A.M., 2012. Interactions between the amnioserosa and the epidermis revealed by the function of the u-shaped gene. Biology open, 1(4), pp.353-361.

Lakhotia, S.C., Mallik, M., Singh, A.K. and Ray, M., 2012. The large noncoding hsr $\omega-n$ transcripts are essential for thermotolerance and remobilization of hnRNPs, HP1 and RNA polymerase II during recovery from heat shock in Drosophila. Chromosoma, 121(1), pp.49-70.

Lall, S., Piano, F. and Davis, R.E., 2005. Caenorhabditis elegans decapping proteins: localization and functional analysis of Dcp1, Dcp2, and DcpS during embryogenesis. Molecular biology of the cell, 16(12), pp.5880-5890.

Lee, G., Kim, K.M., Kikuno, K., Wang, Z., Choi, Y.J. and Park, J.H., 2008. Developmental regulation and functions of the expression of the neuropeptide corazonin in Drosophila melanogaster. Cell and tissue research, 331(3), pp.659-673.

Lin, M.D., Fan, S.J., Hsu, W.S. and Chou, T.B., 2006. Drosophila decapping protein 1, dDcp1, is a component of the oskar mRNP complex and directs its posterior localization in the oocyte. Developmental cell, 10(5), pp.601-613.

Lin, M.D., Jiao, X., Grima, D., Newbury, S.F., Kiledjian, M. and Chou, T.B., 2008. Drosophila processing bodies in oogenesis. Developmental biology, 322(2), pp.276-288.

Liu, Y., Liao, S., Veenstra, J.A. and Nässel, D.R., 2016. Drosophila insulin-like peptide 1 (DILP1) is transiently expressed during non-feeding stages and reproductive dormancy. Scientific reports, 6, p.26620.

Lukacsovich, T., Asztalos, Z., Awano, W., Baba, K., Kondo, S., Niwa, S. and Yamamoto, D., 2001. Dualtagging gene trap of novel genes in Drosophila melanogaster. Genetics, 157(2), pp.727-742.

Mani, M., Chen, C., Amblee, V., Liu, H., Mathur, T., Zwicke, G., Zabad, S., Patel, B., Thakkar, J. and Jeffery, C.J., 2014. MoonProt: a database for proteins that are known to moonlight. Nucleic acids research, 43(D1), pp.D277-D282. 
513

514

515

516

517

518

519

520

521

522

523

524

525

526

527

528

529

530

531

532

533

534

535

536

537

538

539

540

541

542

543

544 Sasikumar, S. and Roy, J.K., 2009. Developmental expression of Rab11, a small GTP-binding protein in 545

Martinez Arias, A., 1993. Development and patterning of the larval epidermis of Drosophila. The development of Drosophila melanogaster, 1, pp.517-608.

McClure, K.D. and Heberlein, U., 2013. A small group of neurosecretory cells expressing the transcriptional regulator apontic and the neuropeptide corazonin mediate ethanol sedation in Drosophila. Journal of Neuroscience, 33(9), pp.4044-4054.

Nandy, N. and Roy, J.K., 2019. Rab11 is essential for lgl mediated JNK-Dpp signaling in dorsal closure and epithelial morphogenesis in Drosophila. bioRxiv, p.713115.

Narasimha, M. and Brown, N.H., 2006. Confocal Microscopy of Drosophilia Embryos. In Cell Biology (pp. 77-86). Academic Press.

Nässel, D.R., Enell, L.E., Santos, J.G., Wegener, C. and Johard, H.A., 2008. A large population of diverse neurons in the Drosophila central nervous system expresses short neuropeptide F, suggesting multiple distributed peptide functions. BMC neuroscience, 9(1), p.90.

Neumann, C.J. and Cohen, S.M., 1997. Long-range action of Wingless organizes the dorsal-ventral axis of the Drosophila wing. Development, 124(4), pp.871-880.

Noselli, S. and Agnès, F., 1999. Roles of the JNK signaling pathway in Drosophila morphogenesis. Current opinion in genetics \& development, 9(4), pp.466-472.

Noselli, S., 1998. JNK signaling and morphogenesis in Drosophila. Trends in Genetics, 14(1), pp.33-38.

Park, D., Veenstra, J.A., Park, J.H. and Taghert, P.H., 2008. Mapping peptidergic cells in Drosophila: where DIMM fits in. PloS one, 3(3), p.e1896.

Rämet, M., Lanot, R., Zachary, D. and Manfruelli, P., 2002. JNK signaling pathway is required for efficient wound healing in Drosophila. Developmental biology, 241(1), pp.145-156.

Rehwinkel, J.A.N., Behm-Ansmant, I., Gatfield, D. and Izaurralde, E., 2005. A crucial role for GW182 and the DCP1: DCP2 decapping complex in miRNA-mediated gene silencing. Rna, 11(11), pp.1640-1647.

Ren, J., Sun, J., Zhang, Y., Liu, T., Ren, Q., Li, Y. and Guo, A., 2012. Down-regulation of Decapping Protein 2 mediates chronic nicotine exposure-induced locomotor hyperactivity in Drosophila. PloS one, 7(12), p.e52521.

Rincón-Limas, D.E., Lu, C.H., Canal, I., Calleja, M., Rodríguez-Esteban, C., Izpisúa-Belmonte, J.C. and Botas, J., 1999. Conservation of the expression and function of apterous orthologs in Drosophila and mammals. Proceedings of the National Academy of Sciences, 96(5), pp.2165-2170.

Santos, J.G., Vömel, M., Struck, R., Homberg, U., Nässel, D.R. and Wegener, C., 2007. Neuroarchitecture of peptidergic systems in the larval ventral ganglion of Drosophila melanogaster. PLoS One, 2(8), p.e695.

5 Drosophila epithelia. Genesis, 47(1), pp.32-39. 
546 Seeger, M., Tear, G., Ferres-Marco, D. and Goodman, C.S., 1993. Mutations affecting growth cone 547 guidance in Drosophila: genes necessary for guidance toward or away from the midline. Neuron, 10(3), 548 pp.409-426.

549 Sha, K., Choi, S.H., Im, J., Lee, G.G., Loeffler, F. and Park, J.H., 2014. Regulation of ethanol-related 550 behavior and ethanol metabolism by the Corazonin neurons and Corazonin receptor in Drosophila 551 melanogaster. PLoS One, 9(1), p.e87062.

552 Shklover, J., Mishnaevski, K., Levy-Adam, F. and Kurant, E., 2016. JNK pathway activation is able to 553 synchronize neuronal death and glial phagocytosis in Drosophila. Cell death \& disease, 6(2), p.e1649.

554 Stronach, B. and Perrimon, N., 2002. Activation of the JNK pathway during dorsal closure in Drosophila 555 requires the mixed lineage kinase, slipper. Genes \& development, 16(3), pp.377-387.

556 Veverytsa, L. and Allan, D.W., 2012. Temporally tuned neuronal differentiation supports the functional 557 remodeling of a neuronal network in Drosophila. Proceedings of the National Academy of Sciences, 558 109(13), pp.E748-E756.

559 Vömel, M. and Wegener, C., 2008. Neuroarchitecture of aminergic systems in the larval ventral ganglion 560 of Drosophila melanogaster. PLoS One, 3(3), p.e1848.

561 Wieschaus, E. and Nüsslein-Volhard, C., 1986. Drosophila: A practical approach. IRL Press, Oxford, 562 England, p.200.

563 Xu, J., Yang, J.Y., Niu, Q.W. and Chua, N.H., 2006. Arabidopsis DCP2, DCP1, and VARICOSE form a 564 decapping complex required for postembryonic development. The Plant Cell, 18(12), pp.3386-3398.

565 Yao, T. and Ndoja, A., 2012, July. Regulation of gene expression by the ubiquitin-proteasome system. 566 In Seminars in cell \& developmental biology (Vol. 23, No. 5, pp. 523-529). Academic Press.

567 Zecca, M., Basler, K. and Struhl, G., 1995. Sequential organizing activities of engrailed, hedgehog and 568 decapentaplegic in the Drosophila wing. Development, 121(8), pp.2265-2278.

569 Zhao, Y., Bretz, C.A., Hawksworth, S.A., Hirsh, J. and Johnson, E.C., 2010. Corazonin neurons function 570 in sexually dimorphic circuitry that shape behavioral responses to stress in Drosophila. PLoS One, 5(2), 571 p.e9141. 


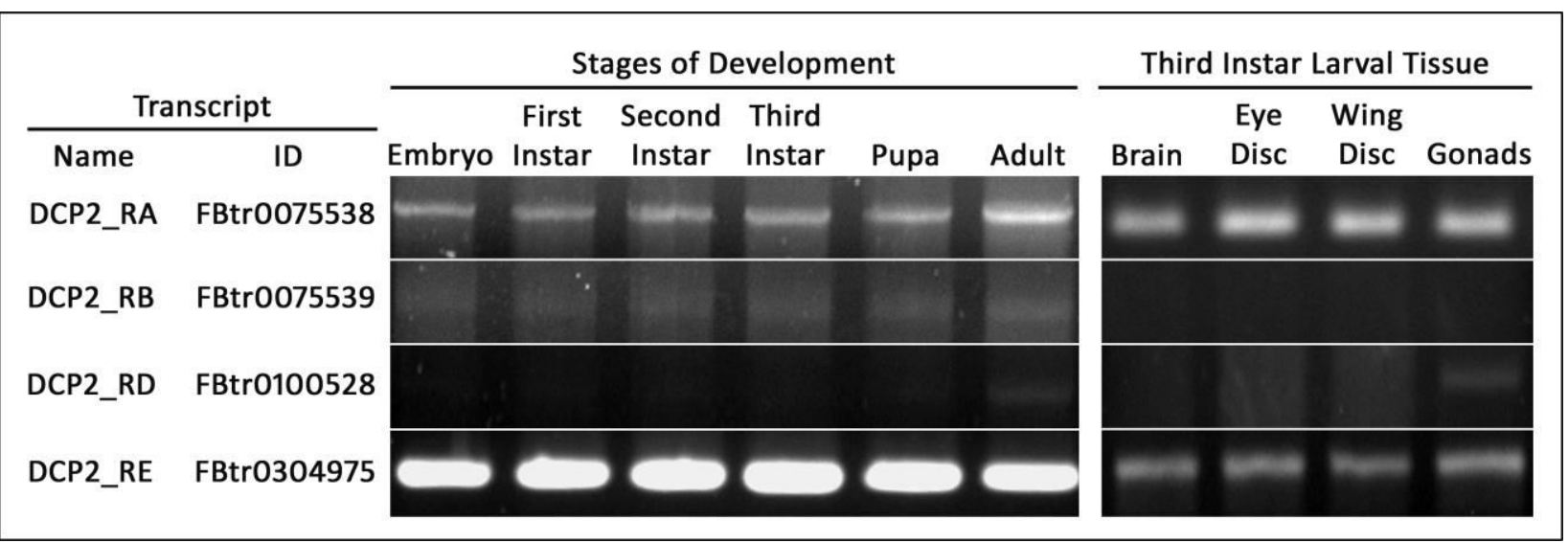

2 Figure 1: Electrophoretogram showing the expression pattern of different isoforms or splice variants of 3 DCP2 across Drosophila development and in selected third instar larval tissues. 


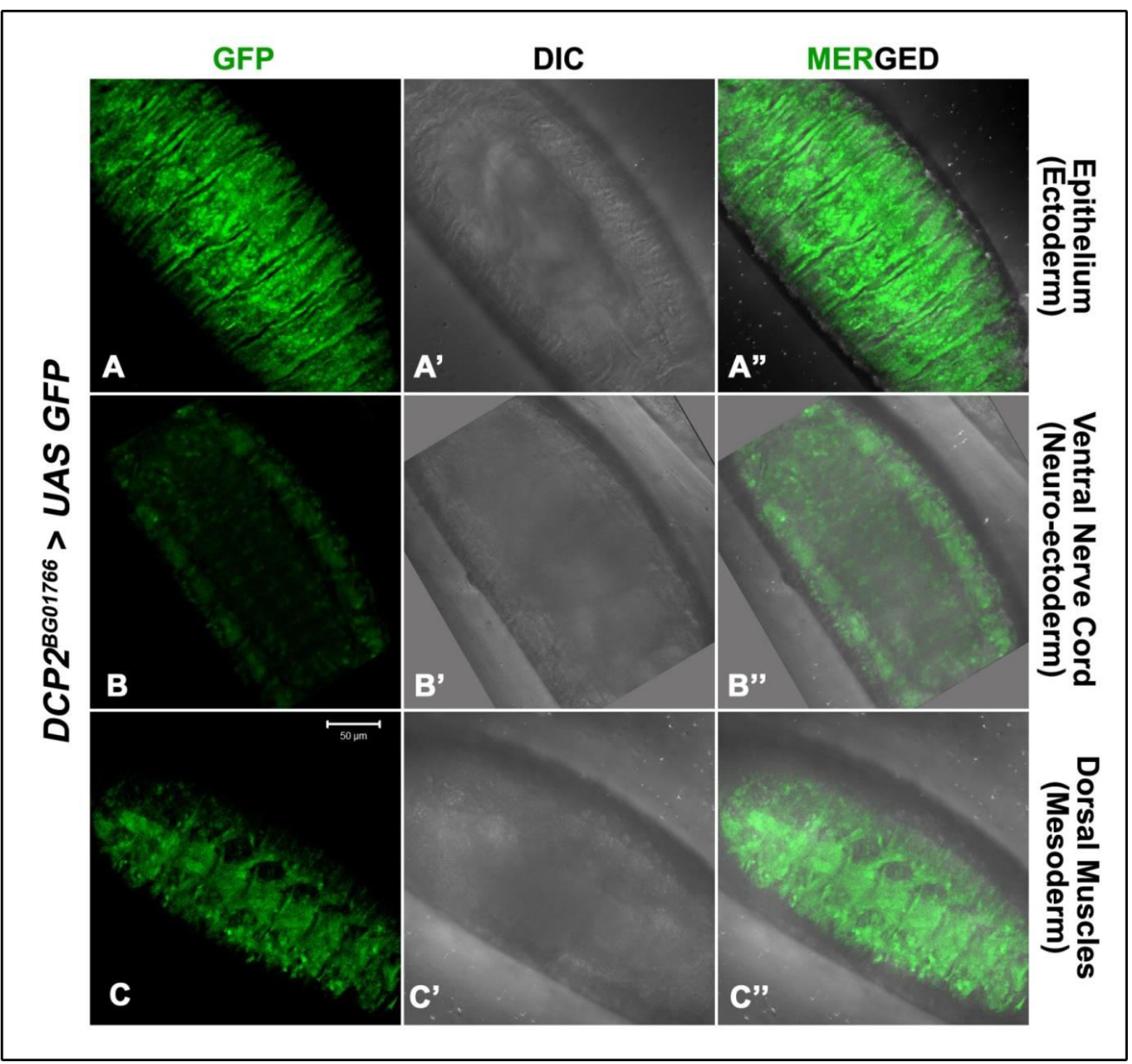

$7 \quad$ Figure 2: Confocal projections of late embryos (Stage 17) showing endogenous expression pattern of $8 D C P 2$ as determined by expression of GFP (green) by $D C P 2^{G A L 4}$. Tissues of differing developmental 9 lineages, viz., ectoderm (A-A"), neuro-ectoderm (B-B") and mesoderm (C-C") show robust expression of 10 GFP. 

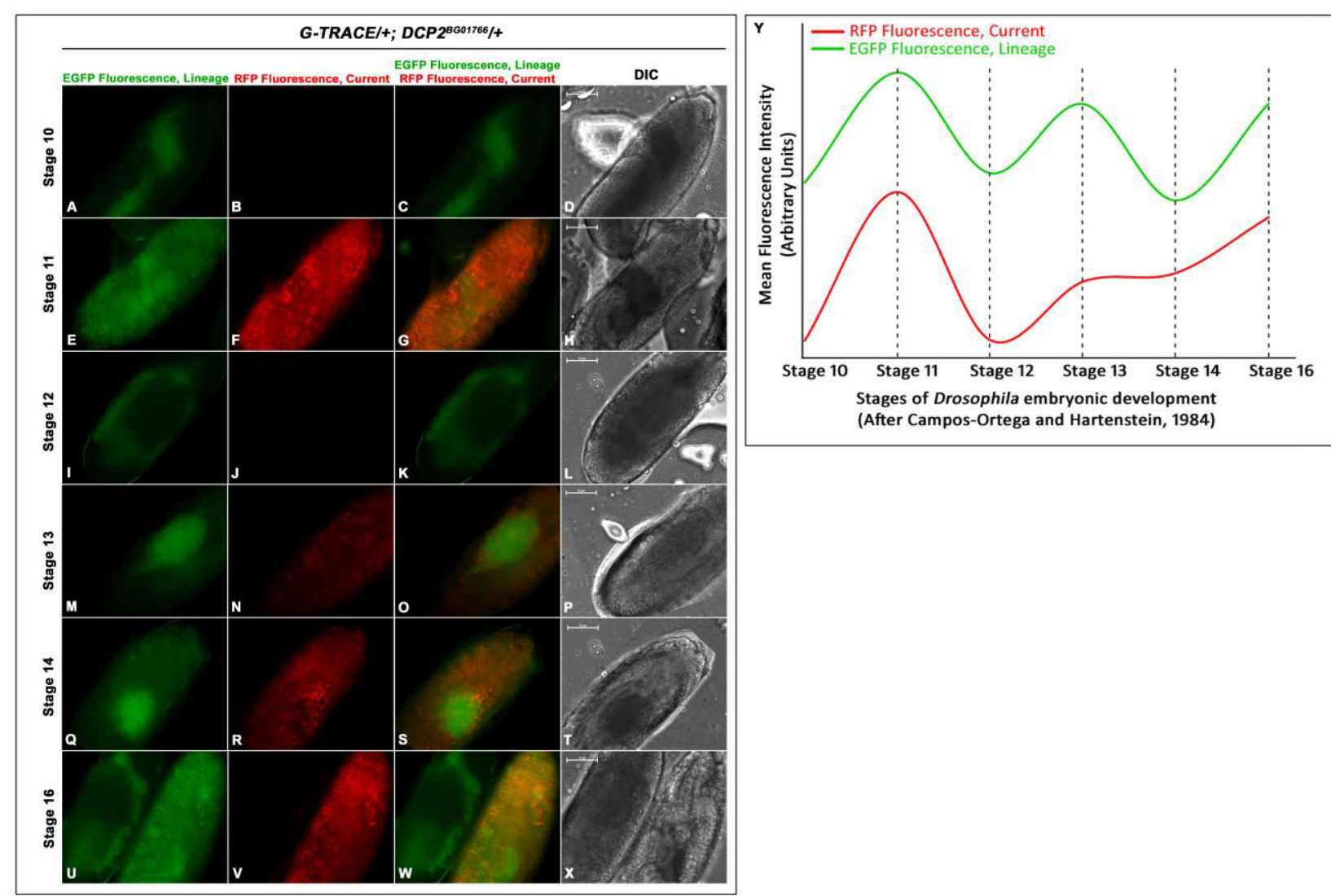

14 Figure 3: Lineage specific (EGFP) and real time (RFP) expression of $D C P 2$ in the embryonic stages 15 using the GAL4-UAS based G-TRACE system. A-X show the expression pattern of the reporters along with the DIC images of the embryos. While real-time $D C P 2$ promoter activity is not detectable during Stages 10 and 12 and is low in Stage 13, it is robust in Stages 11 and 14. Y shows a plot of the fluorescence of both the reporters (GFP and RFP) across the stages observed, wherein a near-sinusoidal curve is obtained showing crests and troughs of $D C P 2$ promoter activity across the stages of Dorsal closure. 


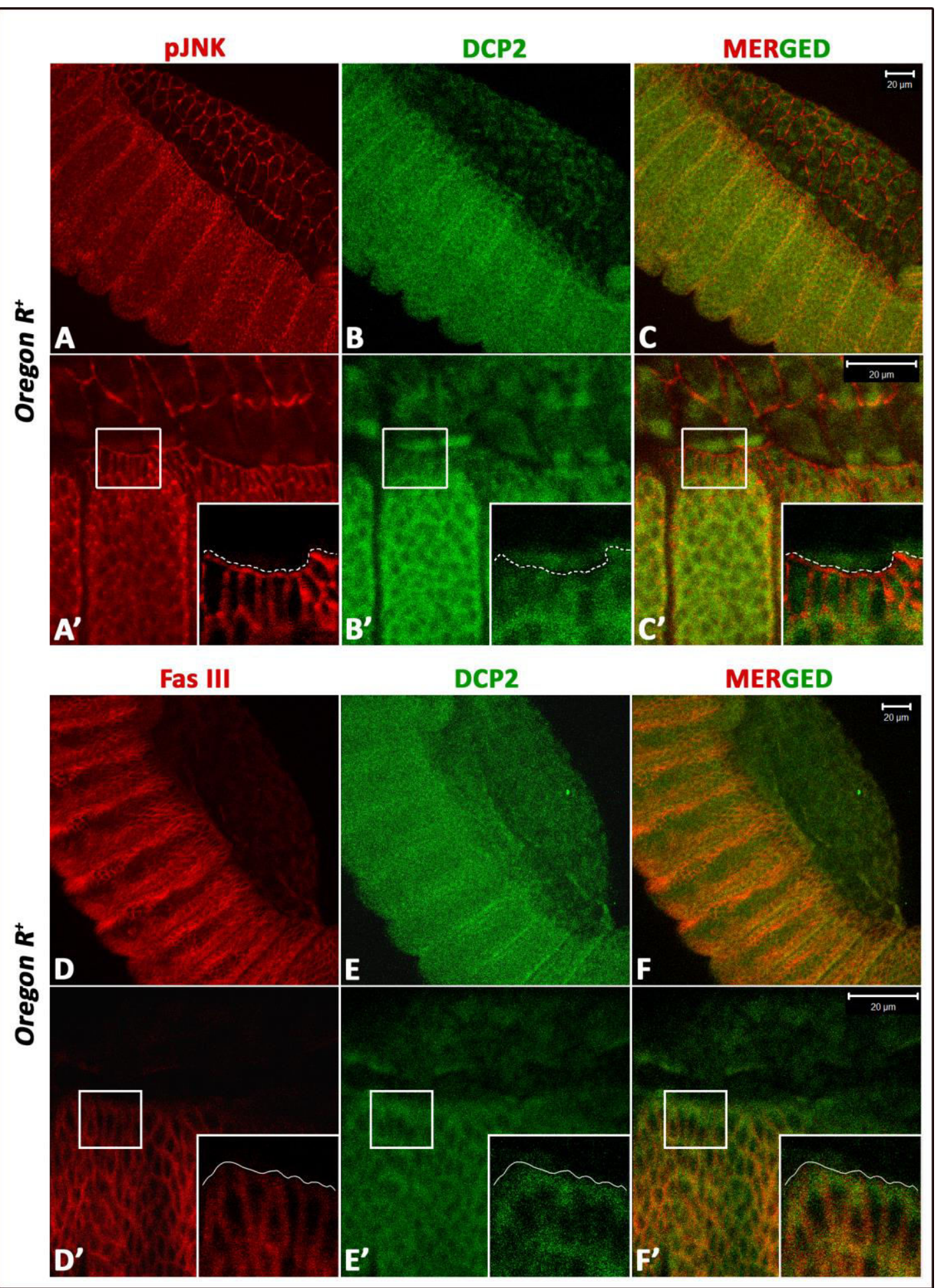

Figure 4: Confocal projections showing immunolocalisation of $\mathrm{DCP} 2$ in the amnioserosa and the lateral 25 epithelium in Stage 13 embryos of wild type strain, co-stained for phospho-JNK (A-C) or the septate 26 junction marker FasIII (D-F). In both cases, punctate expression of DCP2 in the lateral epithelium and 
bioRxiv preprint doi: https://doi.org/10.1101/830729; this version posted November 4, 2019. The copyright holder for this preprint (which was not certified by peer review) is the author/funder. All rights reserved. No reuse allowed without permission.

27 amnioserosa (B and E) and at the leading edge (B' and E') is clearly visible. 


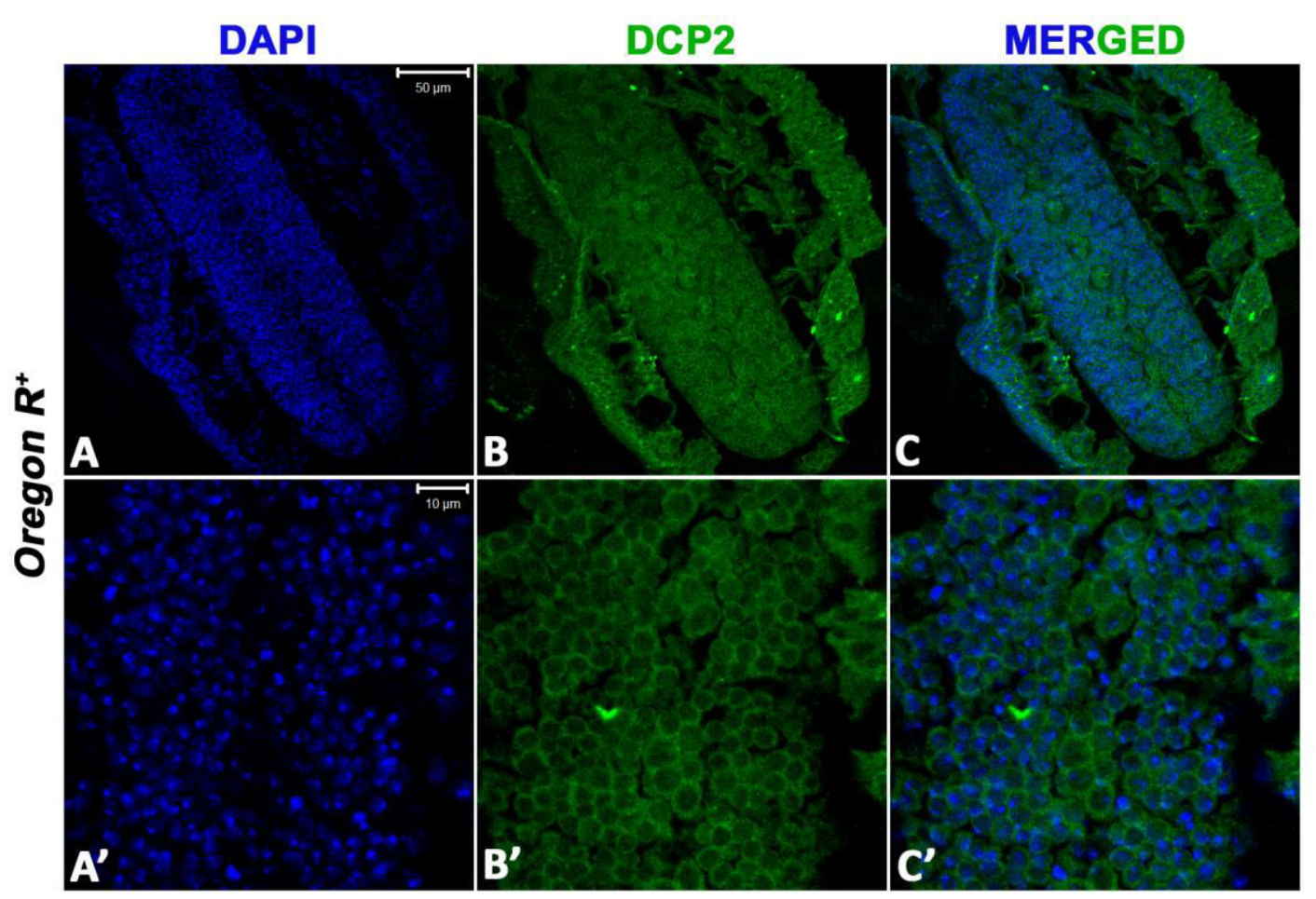

30 Figure 5: Confocal projections showing immunolocalisation of DCP2 in the ventral nerve cord of Stage 3116 embryos of wild type strain show cytoplasmic expression of DCP2 (B and B') in the ventral nerve cord. Nuclei are counterstained with DAPI. 

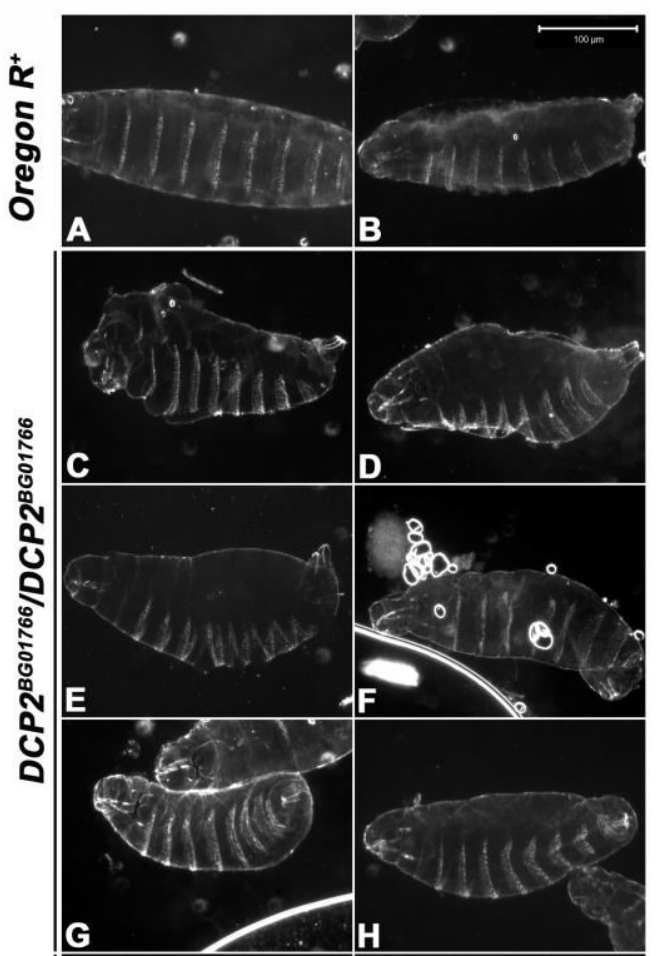

35 Figure 6: Dark field photomicrographs of embryonic cuticles of the wild type (A and B) and DCP2 loss36 of-function homozygotes, viz., $D C P 2^{B G 01766}(\mathrm{C}-\mathrm{H})$ and $D C P 2^{e 00034}(\mathrm{I}-\mathrm{N})$. Note the altered dimensions 37 and /or morphology and defects in head involution exhibited by the mutants as compared to the wild type. 

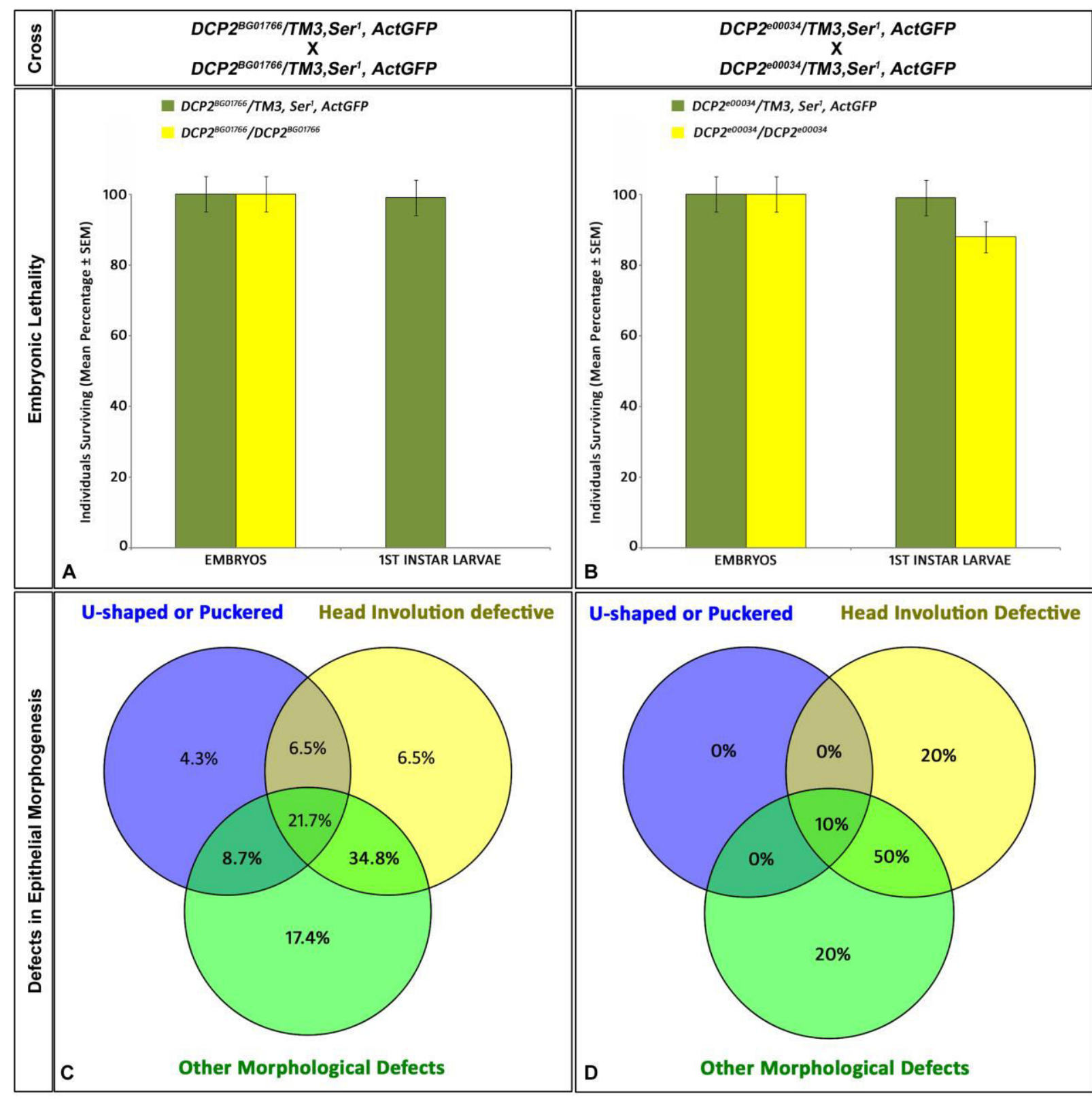

Figure 7: Embryonic Lethality and Defects in Epithelial Morphogenesis in DCP2 loss-of-function homozygotes. $D C P 2^{B G 01766}$ homozygotes are $100 \%$ embryonic lethal (A) and exhibit a broader range of epithelial morphogenesis defects being altered in anterio-posterior or dorso-ventral dimensions along with puckering and defective head involution (C), but $D C P 2^{e 00034}$ homozygotes show only $12 \%$ lethality at the embryonic stage and display a milder range of defects with none of them being exclusively u-shaped or 


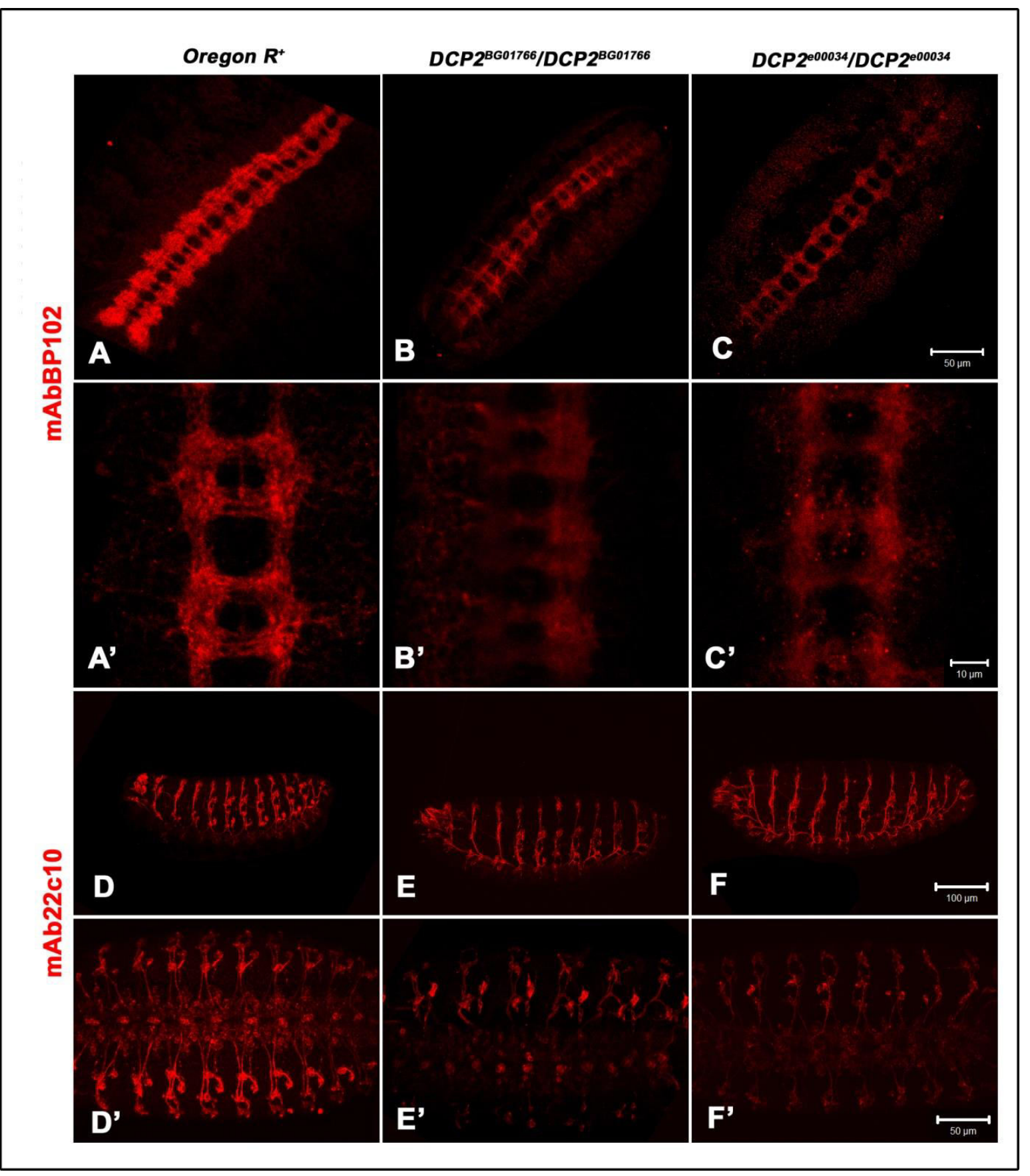

Figure 8: $D C P 2$ null homozygotes display defects in CNS and PNS organization. Upper panel: Wild type embryos, stained with mAbBP102 show regular arrangement of longitudinal connectives and segmental commissures (A and $\mathrm{A}^{\prime}$ ). $D C P 2^{B G 01766}$ homozygotes showed thinning of longitudinal connectives and compressed segmental commissures (B and B'), whereas $D C P 2^{e 00034}$ homozygotes showed thinning of longitudinal connectives and lateral commissures (C and C'). Lower panel: In the PNS, axons run from the ventral nerve cord to the periphery of the embryos in each hemisegment (D and $\left.\mathrm{D}^{\prime}\right)$. A loss of DCP2 causes misrouting and collapse of fasciculating axons (E and $E^{\prime} ; \quad F$ and $F^{\prime}$ ). 


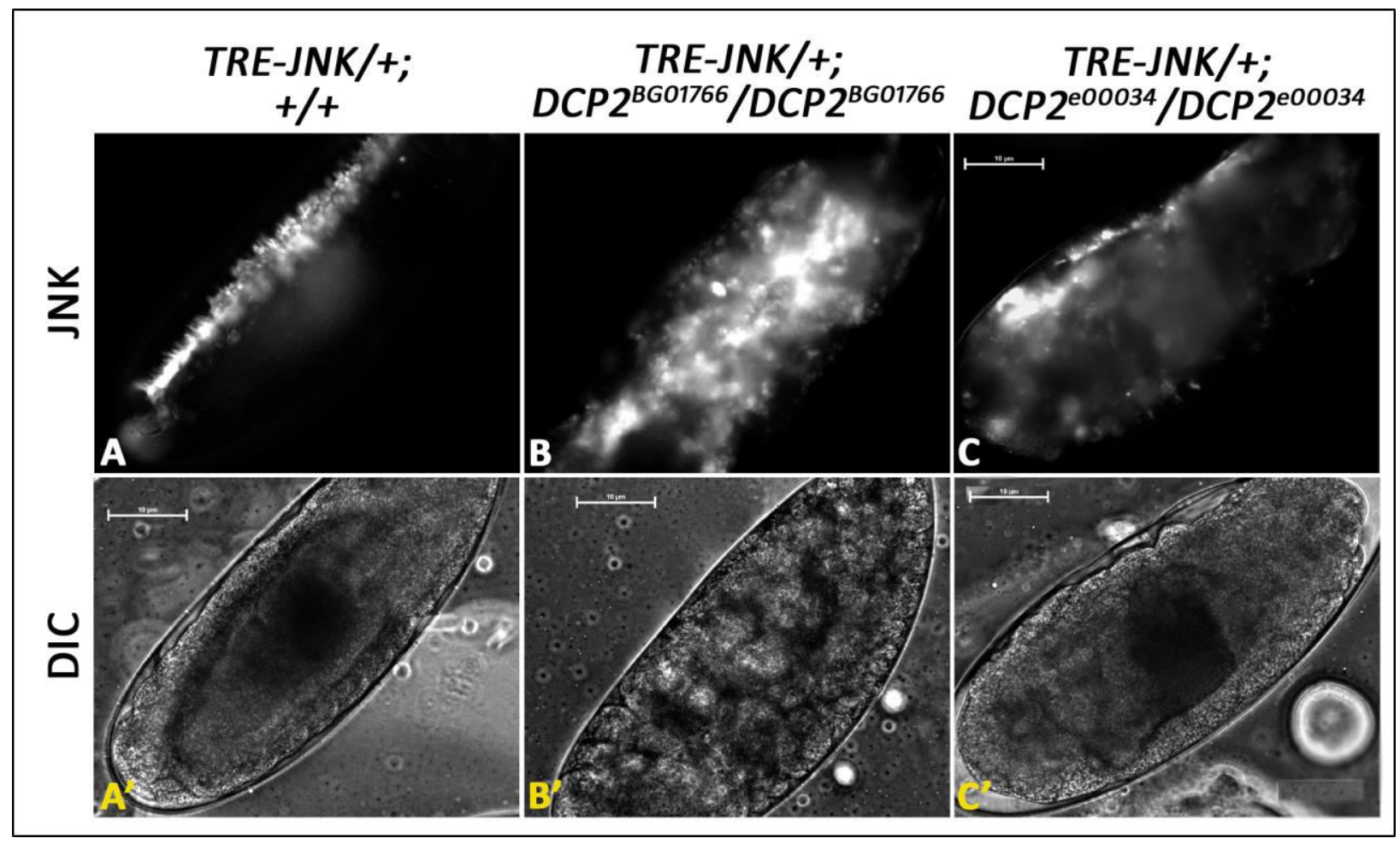

57 Figure 9: Expression of JNK as determined by the biosensor, TRE-JNK in Stage 15 embryos of wild type

58 and DCP2 loss-of-function mutants. While JNK appears as a suture in the wild type embryos (A and A'),

59 it's spatial expression is completely disrupted in DCP2 mutant homozygotes (B and C). 


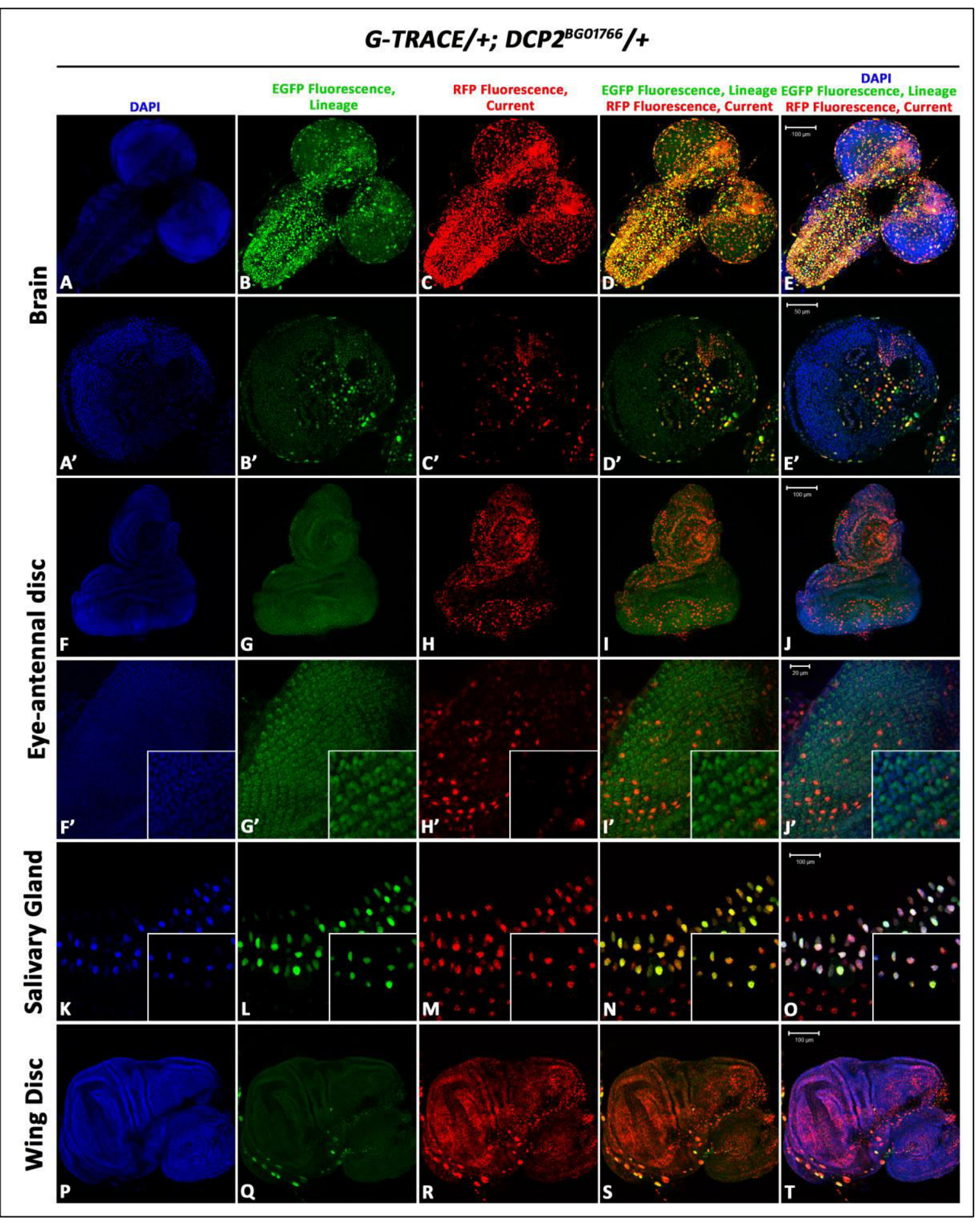

Figure 10: Lineage specific (EGFP) and real time (RFP) expression of $D C P 2$ in the larval tissues using the GAL4-UAS based G-TRACE system. Although the ventral ganglion (B and $C)$ and the antennal disc $(\mathrm{G}$ and $\mathrm{H})$ show significant overlap of the reporters, the central brain ( $\mathrm{B}^{\prime}$ and $\left.\mathrm{C}^{\prime}\right)$ and the eye-disc (G' and $\mathrm{H}^{\prime}$ ) show heterogeneity of expression. The salivary gland nuclei and the wing disc show strong real-time 
bioRxiv preprint doi: https://doi.org/10.1101/830729; this version posted November 4, 2019. The copyright holder for this preprint (which was not certified by peer review) is the author/funder. All rights reserved. No reuse allowed without permission.

66 expression of the DCP2 promoter alongwith prior developmental expression. 


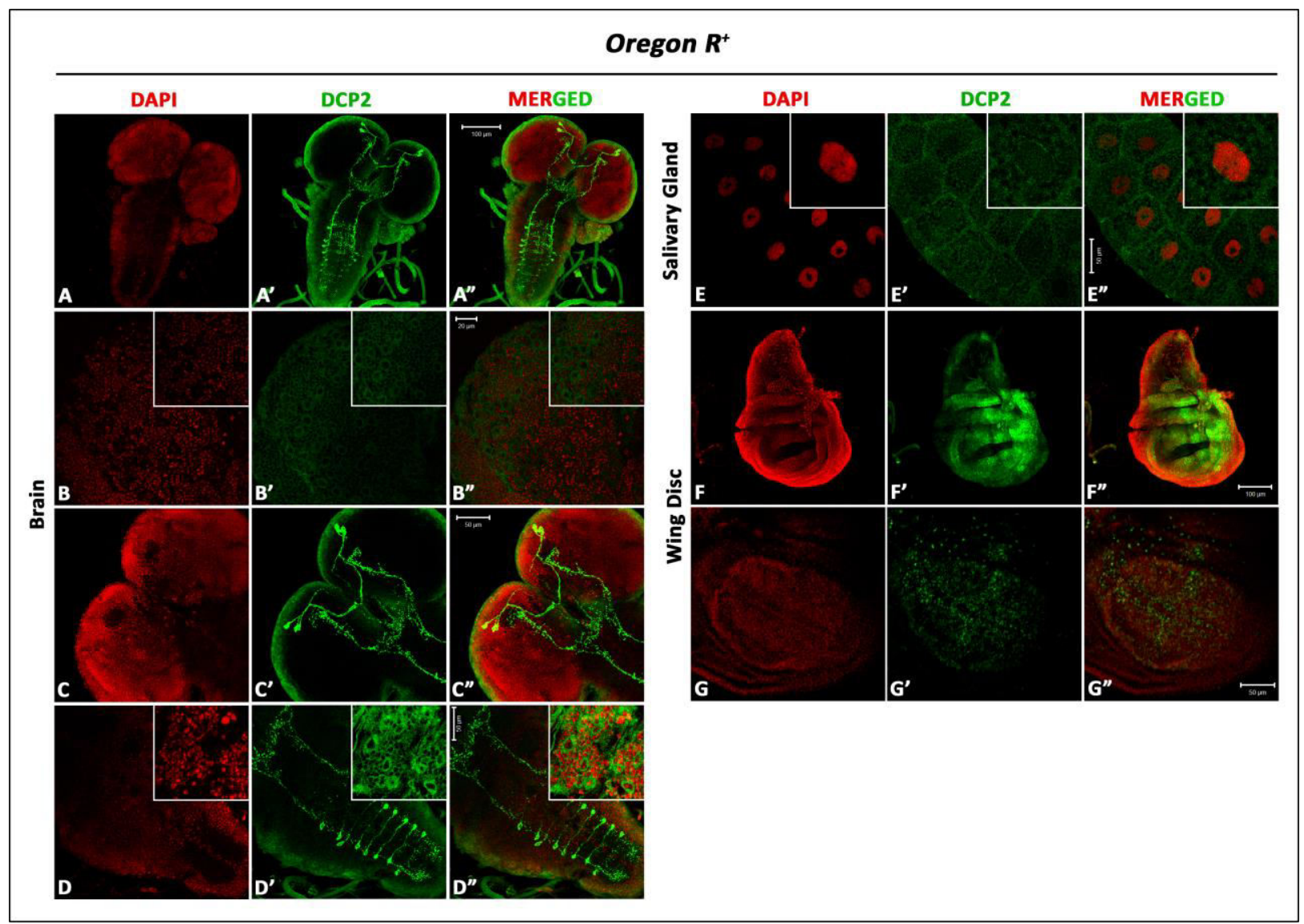

Figure 11: Confocal projections showing immunolocalisation of DCP2 in the larval tissues. A-A" shows

70 the expression pattern in the larval brain. B, C and D show higher magnifications of the same, wherein we

71 find a ubiquitous cytoplasmic expression of DCP2. Visible in C' and D' are a subset of neurons which

72 show high expression of DCP2. In the salivary glands (E), besides cytoplasmic expression, the vesicles in

73 the cytoplasm appear to be arduously decorated with punctate distribution of DCP2. F shows the pattern

74 of expression of DCP2 in the wing disc. Shown in $\mathrm{G}$ is a confocal section which shows a magnified view

75 of the wing pouch wherein DCP2 at the anterio-posterior and dorso-ventral margins in a cruciform 76 pattern. 


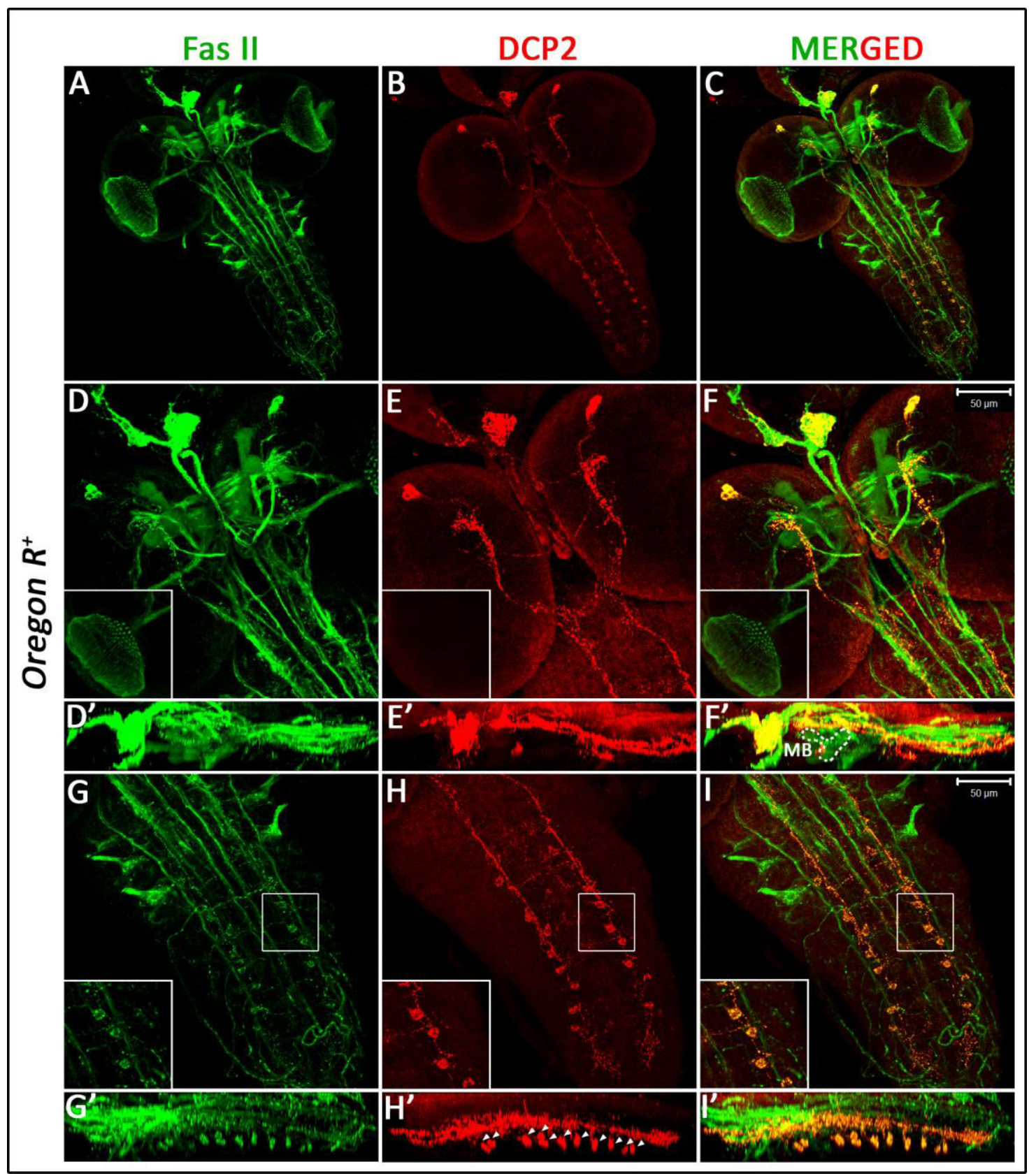

79 Figure 12: Mapping of the neuron(s) expressing high titres of DCP2 in the whole mount preparations of the larval brain (A-C) in the FasII landmark system (Santos et al, 2007). Note the absence of DCP2 in the neurons of the optic lobe (inset D-F). Z-axis stacks show that the DCP2 positive immunopositive neuronal tracts lie below the FasII immunopositive tracts in the larval ventral ganglion but ascend over the Mushroom Body (MB) in the central brain (D'-F'). However, a subset of thoracic and abdominal neuromeres co-express FasII and DCP2 (G-I). Z-axis stacks (G' - I') showing lateral view of the larval ventral ganglion depicted in G-I demonstrate co-expression of FasII and DCP2 in the neuromeres. 


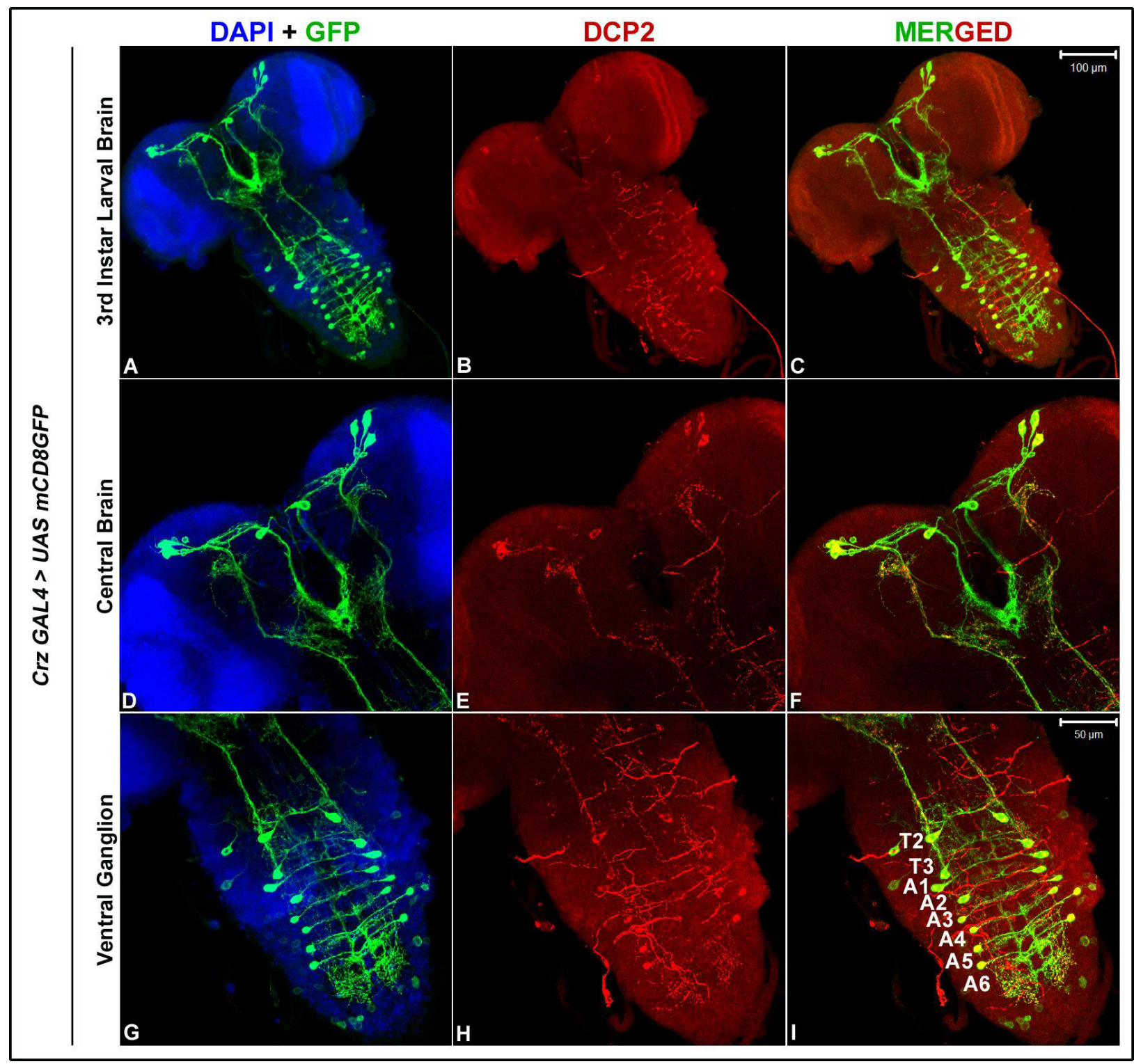

88 Figure 13: Mapping of the neuron(s) expressing DCP2 in the whole mount preparations of the larval

89 brain against the Corazonin expressing neurons. A-C shows the expression pattern of DCP2 and 90 Corazonin neurons in the larval brain. D-F and G-I show magnified view of the central brain and the 91 ventral ganglion respectively, where complete colocalization of DCP2 and Corazonin is visible. 
Male
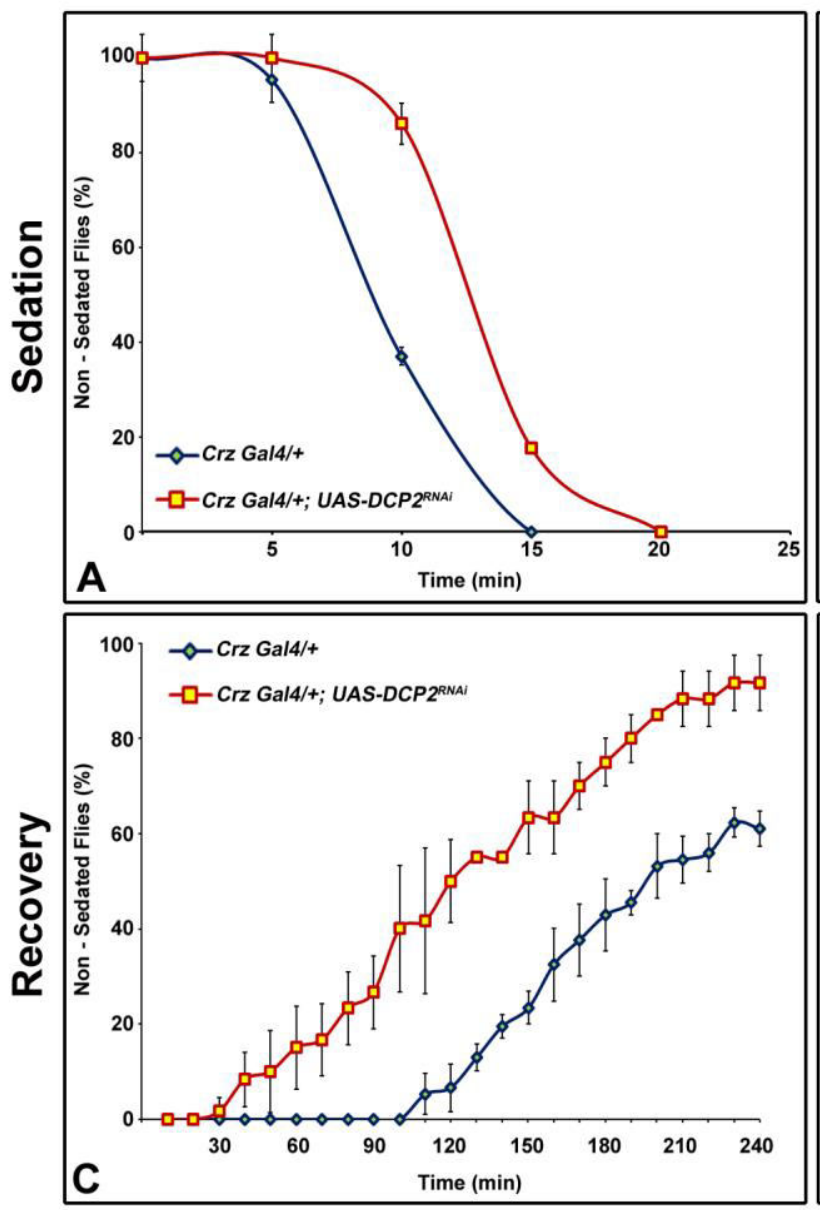

Female
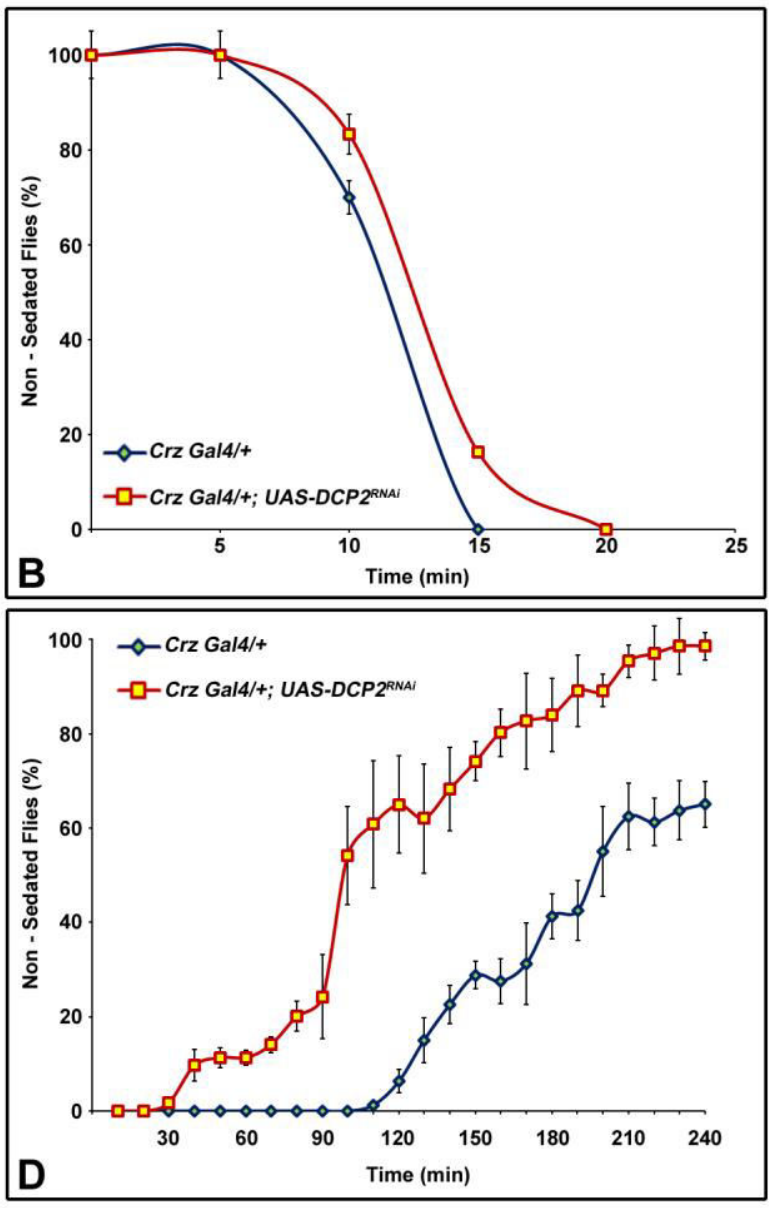

Figure 14: Graphs showing the response to Ethanol induced sedation (A and B) in the control ( $\mathrm{Crz}$ Gal4/+; blue lines) or DCP2 knocked down (Crz-Gal4/+; UAS-DCP2 $2^{R N A i} /+$ ) flies (red lines) and recovery from the same ( $\mathrm{C}$ and $\mathrm{D})$. The knocked-down flies (both male and female) show reduced sensitivity to ethanol vapours as is visible from their delayed sedation behaviour (A and B) or enhanced recovery from sedation (C and D). 